\title{
A Triparametric Family of Optimal Fourth-Order Multiple-Root Finders and Their Dynamics
}

\author{
Young Ik Kim and Young Hee Geum \\ Department of Applied Mathematics, Dankook University, Cheonan 330-714, Republic of Korea \\ Correspondence should be addressed to Young Hee Geum; conpana@empal.com
}

Received 4 September 2015; Revised 26 December 2015; Accepted 4 January 2016

Academic Editor: Juan R. Torregrosa

Copyright ( 2016 Y. I. Kim and Y. H. Geum. This is an open access article distributed under the Creative Commons Attribution License, which permits unrestricted use, distribution, and reproduction in any medium, provided the original work is properly cited.

\begin{abstract}
We investigate the complex dynamics of a triparametric family of optimal fourth-order multiple-root solvers by analyzing their basins of attraction along with extensive study of Möbius conjugacy maps and extraneous fixed points applied to a prototype quadratic polynomial raised to the power of the known integer multiplicity $\mathrm{m}$. A $600 \times 600$ uniform grid centered at the origin covering $6 \times 6$ square region is chosen to display the initial points on each basin of attraction according to a coloring scheme based on their orbit behavior. With illustrative basins of attractions applied to various test polynomials and the corresponding statistical data for convergence as well as a number of comparisons made among the listed methods, we confirm our investigation and analysis developed in this paper.
\end{abstract}

\section{Introduction}

Many researchers [1-7] have shown their interest in the dynamics of iterative methods locating the multiple roots $[8,9]$ of a nonlinear equation. To ensure the convergence of an iterative method in a root-finding problem [10], it is very important to take a good initial value [11-17] close to the desired zero of the given nonlinear equation under consideration. In connection with such a choice of a good initial value, we pay a special attention to the complex dynamics for a number of optimal fourth-order multiple-root finders by investigating their basins of attraction.

Definition 1. Let $\left\{x_{n}\right\}_{n=0}^{\infty}$ be a sequence converging to $\alpha$ and let $e_{n}=x_{n}-\alpha$ be the $n$th iterate error. If there exist real numbers $p$ and a nonzero constant $b$ such that the following error equation holds

$$
e_{n+1}=b e_{n}^{p}+O\left(e_{n}^{p+1}\right)
$$

then $b$ or $|b|$ is called the asymptotic error constant and $p$ is called the order of convergence [18].

Definition 2. Let $d$ be the number of distinct functional or derivative evaluations per iteration. The efficiency index [19] is defined by $\mathrm{EI}=p^{1 / d}$, where $p$ is the order of convergence.

In our study, all the listed methods have the same EI = $4^{1 / 3} \approx 1.587$ agreeing with Kung-Traub optimality [19] conjecture. We investigate the basins of attraction of a number of iterative methods with various test polynomials. Typical fourth-order multiple-root finders developed by Kanwar et al. [20], Soleymani and Babajee [21], and Shengguo et al. [6] are conveniently denoted by Kan, Sol, and Li for later use. Besides, by extending the work of Geum and Kim [22, 23], we propose a triparametric family of optimal fourthorder methods $Y k$ 's whose developments will be described in Section 2. They are listed below in their respective order.

Kan:

$$
y_{n}=x_{n}-\frac{2 m}{m+2} \frac{f\left(x_{n}\right)}{f^{\prime}\left(x_{n}\right)},
$$




$$
\begin{gathered}
x_{n+1}=x_{n}-\frac{m f\left(x_{n}\right)\left\{m^{2}(3 m-2) f^{\prime}\left(y_{n}\right)^{2}-C_{1} f^{\prime}\left(x_{n}\right) f^{\prime}\left(y_{n}\right)+C_{2} f^{\prime}\left(y_{n}\right)^{2}\right\}}{2 f^{\prime}\left(x_{n}\right)\left((m-1) d^{m} f^{\prime}\left(x_{n}\right)-m f^{\prime}\left(x_{n}\right)\right)\left(m f^{\prime}\left(y_{n}\right)\right)\left(m f^{\prime}\left(y_{n}\right)-d^{m}(m+8) f^{\prime}\left(x_{n}\right)\right)}, \\
d=\left(\frac{m}{m+2}\right)^{m}, \text { where } C_{1}=m d\left(6 m^{2}+17 m-14\right) \text { with } C_{2}=d^{2}\left(3 m^{3}+19 m^{2}+16 m+16\right) .
\end{gathered}
$$

Sol:

$$
\begin{aligned}
& y_{n}=x_{n}-\frac{2 m}{m+2} \frac{f\left(x_{n}\right)}{f^{\prime}\left(x_{n}\right)}, \\
& x_{n+1}=x_{n}+\frac{4 m d}{d\left(m^{2}+2 m-4\right)-m^{2} v}[1 \\
& \left.-\frac{m^{3}(m-2)}{16 d^{2}}\left(v-\frac{m+2}{m} d\right)^{2}\right] \cdot \frac{f\left(x_{n}\right)}{f^{\prime}\left(x_{n}\right)}, \\
& v=\frac{f^{\prime}\left(y_{n}\right)}{f^{\prime}\left(x_{n}\right)}, d=\left(\frac{m}{m+2}\right)^{m} .
\end{aligned}
$$

Li:

$$
\begin{aligned}
& y_{n}=x_{n}-\frac{2 m}{m+2} \frac{f\left(x_{n}\right)}{f^{\prime}\left(x_{n}\right)}, \\
& x_{n+1} \\
& \quad=x_{n}
\end{aligned}
$$

$$
\begin{aligned}
& -\frac{(1 / 2) m(m-2) d^{-1} f^{\prime}\left(y_{n}\right)-\left(m^{2} / 2\right) f^{\prime}\left(x_{n}\right)}{f^{\prime}\left(x_{n}\right)-d^{-1} f^{\prime}\left(y_{n}\right)} \\
& \cdot \frac{f\left(x_{n}\right)}{f^{\prime}\left(x_{n}\right)}, \quad d=\left(\frac{m}{m+2}\right)^{m} .
\end{aligned}
$$

$Y k$ :

$$
\begin{aligned}
y_{n}= & x_{n}-\frac{2 m}{m+2} \frac{f\left(x_{n}\right)}{f^{\prime}\left(x_{n}\right)}, \\
x_{n+1}= & y_{n}-\left(\frac{a}{f^{\prime}\left(x_{n}\right)}+\frac{b}{f^{\prime}\left(y_{n}\right)}\right) f\left(x_{n}\right) \\
& -\left(\frac{c}{f^{\prime}\left(x_{n}\right)}+\frac{d}{f^{\prime}\left(y_{n}\right)}\right) F\left(y_{n}\right),
\end{aligned}
$$

where $F\left(y_{n}\right)=f\left(x_{n}\right)+\left(y_{n}-x_{n}\right) \frac{\lambda f^{\prime}\left(x_{n}\right) f^{\prime}\left(y_{n}\right)}{f^{\prime}\left(x_{n}\right)+\rho f^{\prime}\left(y_{n}\right)}$,

where $a, b, c, d, \lambda$, and $\rho$ are parameters to be chosen for fourth order of optimal convergence $[19,24]$. Typical cases of methods $Y k$ 's are presented in Table 1 for $1 \leq k \leq 6$ with selected parameters $\lambda, \rho$, and $d$.

\section{Convergence Analysis}

We describe the main theorem regarding the convergence behavior of proposed family of methods (5) and select parameters $a, b$, and $c$ for the quartic convergence with the aid of Taylor expansion and symbolic computation of Mathematica [25].

Theorem 3. Let $f: \mathbb{C} \rightarrow \mathbb{C}$ have a zero $\alpha$ with integer multiplicity $m \geq 1$ and be analytic in a small neighborhood of $\alpha$. Let $x_{0}$ be an initial guess chosen in a sufficiently small neighborhood of $\alpha$. Let $\kappa=(m /(m+2))^{m}, \gamma=2 m /(m+2)$, and $\lambda, \rho, d \in \mathbb{R}$ be free constant parameters. Let $a=(1 / 8(m+$ 2) $\gamma \kappa \lambda) \tau-m(m+2)^{4} \kappa^{3}(\gamma \lambda-\rho) \rho^{2}+\kappa[8 d(m+2) \gamma \lambda(\gamma \lambda-$ $\left.\rho)+m^{3}\left(-2\left(m^{2}+5 m+8\right) \gamma \lambda+3(m+2)^{2} \rho\right)\right], b=m(m+$ $2)^{3} \kappa(1+\kappa \rho) / 8-d, c=-\left(m^{4}+\kappa\left(3 m^{3}(m+2)-8 d \gamma \lambda\right) \rho+\right.$ $\left.3 m^{2}(m+2)^{2} \kappa^{2} \rho^{2}+m(m+2)^{3} \kappa^{3} \rho^{3}\right) / 8 \gamma \kappa \lambda$, and $\tau=m^{4}(m+2)-$ $3 m^{2}(m+2)^{3} \kappa^{2}(\gamma \lambda-\rho) \rho$. Then iterative method (5) is of order four and defines a triparametric family of iterative methods with the following error equation:

$$
e_{n+1}=\psi_{4} e_{n}^{4}+O\left(e_{n}^{5}\right), \quad n=0,1,2, \ldots
$$

where $\psi_{4}=\left(\left(m\left(8+2 m+6 m^{2}+4 m^{3}+m^{4}\right)+\left(-8+12 m+14 m^{2}+\right.\right.\right.$ $\left.\left.\left.14 m^{3}+6 m^{4}+m^{5}\right) \kappa \rho\right) / 3 m^{4}(m+1)^{3}(m+2)[m+(m+2) \kappa \rho]\right) \theta_{1}^{3}-$ $\left(1 / m(m+1)^{2}(m+2)\right) \theta_{1} \theta_{2}+\left(m /(m+2)^{3}(m+1)(m+3)\right) \theta_{3}$, $\theta_{j}=f^{(m+\mathrm{j})}(\alpha) / f^{(m)}(\alpha)$ for $j \in \mathbb{N}$, and $e_{n}=x_{n}-\alpha$.

Proof. Using Taylor's series about $\alpha$, we have

$$
\begin{aligned}
& f\left(x_{n}\right) \\
& \quad=e^{m} \beta\left(1+A_{1} e+A_{2} e^{2}+A_{3} e^{3}+A_{4} e^{4}+O\left(e_{n}^{5}\right)\right), \\
& f^{\prime}\left(x_{n}\right) \\
& \quad=e^{m-1} \beta m\left(1+B_{1} e+B_{2} e^{2}+B_{3} e^{3}+B_{4} e^{4}+O\left(e_{n}^{5}\right)\right),
\end{aligned}
$$

where $\beta=f^{(m)}(\alpha) / m !, A_{k}=(m ! /(m+k) !) \theta_{k}, B_{k}=((m-$ $1) ! /(m+k-1) !) \theta_{k}$, and $\theta_{k}=f^{(m+k)}(\alpha) / f^{(m)}(\alpha)$ for $k \in \mathbb{N}$.

Dividing (7) by (8) gives us

$$
\frac{f\left(x_{n}\right)}{f^{\prime}\left(x_{n}\right)}=\frac{1}{m}\left[e_{n}-K_{1} e_{n}^{2}-K_{2} e_{n}^{3}+K_{3} e_{n}^{4}+O\left(e_{n}^{5}\right)\right],
$$

where $K_{1}=-A_{1}+B_{1}, K_{2}=-A_{2}+A_{1} B_{1}-B_{1}^{2}+B_{2}$, and $K_{3}=-A_{3}+A_{2} B_{1}-A_{1} B_{1}^{2}+B_{1}^{3}+A_{1} B_{2}-2 B_{1} B_{2}+B_{3}$. 
TABLE 1: Typical methods with selected parameters $(\lambda, \rho)$ and constants $(a, b, c, d)$.

\begin{tabular}{|c|c|c|}
\hline $\mathrm{MT}^{*}$ & $(\lambda, \rho)$ & $(a, b, c, d)$ \\
\hline$Y 1$ & $(-1 / \kappa, 5)$ & $\begin{array}{c}a=\frac{m^{3}\left[m(m+2)+5\left(m^{2}+2 m-4\right) \kappa\right]}{40 \delta}, c=0, d=-\frac{1}{80}(m+2)(m+5 \delta)^{3}, \\
b=\frac{1}{80}(m+2)\left[m^{3}+5 m(4+5 m) \delta+125 m \delta^{2}+125 \delta^{3}\right] .\end{array}$ \\
\hline$Y 2$ & $(1,5)$ & $\begin{array}{c}a=\frac{m^{3}\left[m(m+2)+5\left(m^{2}+2 m-4\right) \kappa\right]}{40 \delta}, c=0, d=\frac{m(m+5 \delta)^{3}}{80 \kappa}, \\
b=\frac{(m+2)\left[-m^{3}-15 m^{2} \delta-65 m \delta^{2}-25 \delta^{2}(10+3 m) \kappa\right]}{80 \kappa} .\end{array}$ \\
\hline$Y 3$ & $(-1,1)$ & $\begin{array}{c}a=-\frac{m^{3}(m+2)^{2}+(2+3 m)^{2} \delta^{3}+m^{2}\left(24+68 m+38 m^{2}+7 m^{3}\right) \kappa+5 m(m+2)(2+3 m) \delta^{2}}{16 \delta}, \\
b=0, c=\frac{(m+2)\left[m\left(m^{2}+3 m \delta+5 \delta^{2}\right)+(2+3 m) \delta^{2} \kappa\right]}{16 \kappa}, d=\frac{1}{8} m(m+2)^{2} \delta(1+\kappa) .\end{array}$ \\
\hline Y4 & $(-6,-2)$ & $\begin{array}{c}a=-\frac{m^{3}(m+2)^{2}+12 m(m+2)(-2+5 m) \delta^{2}-8(2-5 m)^{2} \delta^{3}+6 m^{2}\left(-8+20 m+14 m^{2}+3 m^{3}\right) \kappa}{96 \delta} \\
b=0, c=\frac{(m+2)\left[m^{3}-6 m^{2} \delta-12 m \delta^{2}+8 \delta^{2}(-2+5 m) \kappa\right]}{96 \kappa}, d=\frac{1}{8} m(m+2)^{2} \delta(1+\kappa \rho) .\end{array}$ \\
\hline$Y 5$ & $(-1,0)$ & $a=\frac{m^{3}\left[-(m+2)^{2}-4\left(8+5 m+m^{2}\right) \kappa\right]}{16 \delta}, b=\frac{1}{8} m(m+2)^{2} \delta, c=\frac{m^{3}(m+2)}{16 \kappa}, d=0$ \\
\hline$Y 6$ & $(1 / \kappa, 1)$ & $\begin{array}{c}a=\frac{-m^{3}\left(28+16 m+3 m^{2}\right)-3 m^{2}(m+2)^{2} \delta+m(m+2)^{2} \delta^{2}+(m+2)^{2} \delta^{3}}{16(m+2)} \\
b=\frac{1}{8} m(m+2)^{2} \delta(1+\kappa), c=-\frac{1}{16}(m+2)(m+\delta)^{3}, d=0\end{array}$ \\
\hline
\end{tabular}

${ }^{*}$ MT: method, $\delta=(m+2) \kappa, \kappa=(m /(m+2))^{m}$.

Letting $t=1-\gamma / m$ with the above relation (9), we have

$$
\begin{aligned}
& y_{n}=x_{n}-\gamma \frac{f\left(x_{n}\right)}{f^{\prime}\left(x_{n}\right)}=\alpha+t e+K_{1}(1-t) e_{n}^{2}+K_{2}(1 \\
&-t) e_{n}^{3}+K_{3}(1-t) e_{n}^{4}+O\left(e_{n}^{5}\right) \\
& f^{\prime}\left(y_{n}\right)=m t^{m-1}+m t^{m}\left(B_{1}+\frac{K_{1}(m-1+t-m t)}{t^{2}}\right) \\
& \quad \cdot+\frac{1}{2} m t^{m-3}\left(K_{1}^{2}(m-2)(m-1)(t-1)^{2}\right. \\
& \quad-2 B_{1} K_{1} m(t-1) t^{2}+2 t\left(B_{2} t^{3}+K_{2}(m-1+t\right. \\
&\quad-m t))) e_{n}^{2}+\frac{1}{6} m t^{m-4}\left(-K_{1}^{3}(m-3)(m-2)(m\right. \\
&-1)(t-1)^{3}+3 B_{1} K_{1}^{2}(m-1) m(t-1)^{2} t^{2} \\
& \quad-6 K_{1}(t-1) t\left(-t K_{2}(m-2)(m-1)(t-1)\right. \\
&\left.+B_{2}(m+1) t^{3}\right)+6 t^{2}\left(K_{3}(m-1+t-m t)\right. \\
&\left.\left.+t\left(-B_{1} K_{2} m(t-1)+B_{3} t^{3}\right)\right)\right) e_{n}^{3}+\frac{1}{24} \\
&+m t^{m-5}\left(K_{1}^{4}(m-4)(m-3)(m-2)(m-1)(t\right. \\
&+1)^{4}-4 B_{1} K_{1}^{3}(m-2)(m-1) m(t-1)^{3} t^{2} \\
&+1 K^{2} t\left(-K_{2}(m-3)(m-2)(m-1)(t\right.
\end{aligned}
$$

$$
\begin{aligned}
& \left.-1)+B_{2} m(m+1) t^{3}\right)-24 K_{1}(t-1) t^{2}\left(-K_{3}(m\right. \\
& -2)(m-1)(t-1)+t\left(B_{3}(m+2) t^{3}\right. \\
& \left.\left.+B_{1} K_{2} m(m-1+t-m t)\right)\right)+12 t^{2}\left(K_{2}^{2}(m-2)\right. \\
& \cdot(m-1)(t-1)^{2}-2 B_{2} K_{2}(m+1)(t-1) t^{3} \\
& +2 t\left(K_{4}(m-1)(t-1)\right. \\
& \left.\left.\left.+t\left(-B_{1} K_{3} m(t-1)+B_{4} t^{4}\right)\right)\right)\right) e^{4}+O\left(e_{n}^{5}\right) .
\end{aligned}
$$

Substituting (7)-(10) into (5), we get the error equation:

$$
e_{n+1}=\psi_{1} e_{n}+\psi_{2} e_{n}^{2}+\psi_{3} e_{n}^{3}+\psi_{4} e_{n}^{4}+O\left(e_{n}^{5}\right)
$$

where $\psi_{1}=t-\left(a\left(t+t^{m} \rho\right)+t^{-m}\left(b t\left(t+t^{m} \rho\right)+(d t+\right.\right.$ $\left.\left.\left.c t^{m}\right)\left(t+t^{m}(-\gamma \lambda+\rho)\right)\right)\right) / m\left(t+t^{m} \rho\right)$ and coefficients $\psi_{i}(i=$ $2,3,4)$ depend on the parameters $t, a, b, c, d, \lambda$, and $\rho$ and the function $f(x)$.

Solving $\psi_{1}=0$ and $\psi_{2}=0$ for $a$ and $b$, we have

$$
\begin{aligned}
a= & t\left(m-(b+d) t^{-m}\right) \\
& +\frac{d t \gamma \lambda-c\left(t+t^{m}(-\gamma \lambda+\rho)\right)}{t+t^{m} \rho}
\end{aligned}
$$




$$
\begin{aligned}
b= & -\frac{m t^{m}}{(t-1)(1+m(t-1)+t)} \\
& -\frac{d t^{2}+2 d t^{1+m} \rho+t^{2 m}(c \gamma \lambda+d \rho(-\gamma \lambda+\rho))}{\left(t+t^{m} \rho\right)^{2}} .
\end{aligned}
$$

After substituting $a$ and $b$ into $\psi_{3}=\psi_{31} \theta_{1}^{2}+\psi_{32} \theta_{2}$, we solve $\psi_{3}=0$. Due to the fact that $\psi_{3}$ is independent of $\theta_{1}$ and $\theta_{2}$, solving $\psi_{31}=\psi_{32}=0$ for $c$ and $t$, we have

$$
t=\frac{m}{m+2}
$$

$$
\begin{aligned}
c= & -\frac{1}{2} t^{-m}\left(t+t^{m} \rho\right)^{3} \Omega_{1}\left[2 m t(2+t)+\frac{\Omega_{2}}{t}\right. \\
& \left.+m^{2}\left(\frac{1}{t}-3+4 t^{2}\right)-\frac{2 d t^{m} \rho \Omega_{1}}{\left(t+t^{m} \rho\right)^{3}}\right],
\end{aligned}
$$

where $\Omega_{1}=(t-1)^{2}(1+m(t-1)+t)^{3} \gamma \lambda$ and $\Omega_{2}=m^{3}(t-$ 1) $(1+2(t-1) t)$.

Applying $t=m /(m+2)$ into (12)-(14) with $\gamma=2 m /(m+2)$ and $\kappa=(m /(m+2))^{m}$, we have the following relations:

$$
\begin{aligned}
a= & \frac{1}{8(m+2) \gamma \kappa \lambda} m^{4}(m+2)-3 m^{2}(m+2)^{3} \kappa^{2}(\gamma \lambda-\rho) \rho-m(m+2)^{4} \kappa^{3}(\gamma \lambda-\rho) \rho^{2} \\
& +\kappa\left(8 d(m+2) \gamma \lambda(\gamma \lambda-\rho)+m^{3}\left(-2(8+m(5+m)) \gamma \lambda+3(m+2)^{2} \rho\right)\right), \\
b= & \frac{1}{8}\left(-8 d+m(m+2)^{3} \kappa(1+\kappa \rho)\right), \\
c= & -\frac{m^{4}+\kappa\left(3 m^{3}(m+2)-8 d \gamma \lambda\right) \rho+3 m^{2}(m+2)^{2} \kappa^{2} \rho^{2}+m(m+2)^{3} \kappa^{3} \rho^{3}}{8 \gamma \kappa \lambda} .
\end{aligned}
$$

Thanks to symbolic computation of Mathematica [25], we reach the error equation below:

$$
e_{n+1}=\psi_{4} e_{n}^{4}+O\left(e_{n}^{5}\right)
$$

where $\psi_{4}=\psi_{41} \theta_{1}^{3}+\psi_{42} \theta_{1} \theta_{2}+\psi_{43} \theta_{3}$ with

$$
\begin{aligned}
& \psi_{41}=\frac{m\left(8+2 m+6 m^{2}+4 m^{3}+m^{4}\right)+\left(-8+12 m+14 m^{2}+14 m^{3}+6 m^{4}+m^{5}\right) \kappa \rho}{3 m^{4}(m+1)^{3}(m+2)[m+(m+2) \kappa \rho]} \\
& \psi_{42}=-\frac{1}{m(m+1)^{2}(m+2)}, \\
& \psi_{43}=\frac{m}{(m+2)^{3}(m+1)(m+3)}
\end{aligned}
$$

completing the proof.

Remark 4. If $\lambda=-1 / \kappa \gamma, \rho=-1 / \kappa$, and $d=0$ are selected, then we find relations:

$$
\begin{aligned}
& a=-\frac{m^{3}}{2(m+2)}, \\
& b=0, \\
& c=-m .
\end{aligned}
$$

In this case, proposed method (5) reduces to method Li given by (4).

\section{Conjugacy Maps and Dynamics}

Multipoint iterative methods [19] solving a nonlinear equation of the form $f(x)=0$ can be generally written as a discrete dynamical system

$$
x_{n+1}=R_{f}\left(x_{n}\right),
$$

where $R_{f}$ is the iteration function. We begin by writing (5) in the form of a complex discrete dynamical system:

$$
z_{n+1}=R_{f}\left(z_{n}\right)=z_{n}-\frac{f\left(z_{n}\right)}{f^{\prime}\left(z_{n}\right)} H_{f}\left(z_{n}\right),
$$

where $H_{f}\left(z_{n}\right)=\gamma+(a+c \sigma)+(b+d \sigma)(v / s), \gamma=2 m /(m+2), \sigma=$ $1-\gamma \lambda s /(v+\rho s), s=f\left(z_{n}\right) / f^{\prime}\left(z_{n}\right)$, and $v=f\left(z_{n}\right) / f^{\prime}\left(y_{n}\right) ; a, b$, 
and $c$ are given, respectively, by (12), (13), and (14); $\lambda, \rho, d \in \mathbb{C}$ are free parameters.

Definition 5. Let $F: X \rightarrow X$ and $G: Y \rightarrow Y$ be two functions (dynamical systems). One says that $F$ and $G$ are conjugate if there is a function $h: X \rightarrow Y$ such that $h \circ F=G \circ h$. Then the map $h$ is called a conjugacy [26].

Remark 6. Note that a conjugacy indeed preserves the dynamical behavior between the two dynamical systems; for example, if $F$ is conjugate to $G$ via $h$ and $\xi$ is a fixed point of $F$, then $h(\xi)$ is a fixed point of $G$.

Furthermore, if $h$ is a homeomorphism, that is, if $F$ is topologically conjugate to $G$ via $h$, and $\zeta$ is a fixed point of $G$, then $h^{-1}(\zeta)$ is a fixed point of $F$. Also, we find $G=h \circ F \circ h^{-1}$ and $G^{n}=\left(h \circ F \circ h^{-1}\right) \circ\left(h \circ F \circ h^{-1}\right) \circ \cdots \circ\left(h \circ F \circ h^{-1}\right)=h \circ F^{n} \circ h^{-1}$. If $F$ and $G$ are invertible, then the topological conjugacy $h$ maps an orbit

$$
\ldots, F^{-2}(x), F^{-1}(x), x, F(x), F^{2}(x), \ldots
$$

of $F$, onto an orbit

$$
\ldots, G^{-2}(y), G^{-1}(y), y, G(y), G^{2}(y), \ldots,
$$

of $G$, where $y=h(x)$ and the order of points is preserved. Hence, the orbits of the two maps behave similarly under homeomorphism $h$ or $h^{-1}$.

Via Möbius conjugacy map $M(z)=(z-A) /(z-B), z, A \neq$ $B, A, B \in \mathbb{C} \cup\{\infty\}$, considered by Blanchard [27], $R_{f}$ in (20) is conjugated to $J$ satisfying

$$
J(z ; A, B, \lambda, \rho, d)=\frac{H(z ; A, B, \lambda, \rho, d)}{D(z ; A, B, \lambda, \rho, d)},
$$

when applied to a quadratic polynomial $f(z)=[(z-$ $A)(z-B)]^{m}$ raised to the power of $m$, where $H$ and $D$ are polynomials with no common factors whose coefficients are generally dependent upon parameters $A, B, \lambda$, $\rho$, and $d$. The following theorem favorably indicates that $J$ is dependent only on $\rho$ but independent of parameters $A, B, \lambda$, and $d$.

Theorem 7. Let $f(z)=[(z-A)(z-B)]^{m}$ with $m \in \mathbb{N}$ and $M(z)=(z-A) /(z-B), A \neq B, A, B \in \mathbb{C} \cup\{\infty\}$. Then $R_{f}(z ; \lambda, \rho, d)$ is conjugate to $J(z ; \rho)$ satisfying

$$
J(z ; \rho)=z \cdot \frac{r_{1} \mu+(1+\kappa \rho) \tau-r_{2} \omega}{r_{3} \mu+z(1+\kappa \rho) \tau-r_{4} \omega},
$$

where $r_{1}=m^{3}+\left(m^{3}-4 m-8 z\right) \kappa \rho, r_{2}=4(z-\kappa \rho)+$ $m[2+m(m+3)(1+\kappa \rho)], r_{3}=-8 \kappa \rho+z\left[m^{3}+(m-\right.$ 2) $m(m+2) \kappa \rho], r_{4}=4+(m+2) z[m(m+1)+(m-$ 1) $(m+2) \kappa \rho], \mu=\beta_{1}^{2 m-2} \beta_{2}^{2 m-2} \beta^{2}, \tau=m^{4 m-2}(m+2)^{3}(z+$ 1) ${ }^{4 m} \kappa^{2}, \omega=2 m^{2 m-1}(z+1)^{2 m} \beta_{1}^{m-1} \beta_{2}^{m-1} \beta \kappa, \beta_{1}=m z+m-$ $\gamma, \beta_{2}=m+z(m-\gamma), \beta=m(z+1)^{2}-2 z \gamma, \kappa=(m /(m+2))^{m}$, and $\gamma=2 m /(m+2)$.
Proof. Since the inverse of $M(z)$ is easily found to be $M^{-1}(z)=(B z-A) /(z-1)$, we find after a lengthy computation with the aid of Mathematica [25] symbolic capability:

$$
J(z ; \rho)=M \circ R_{f} \circ M^{-1}(z)=z \cdot \frac{\mathscr{H}(z ; \rho)}{\mathscr{D}(z ; \rho)},
$$

where $\mathscr{H}(z ; \rho)=r_{1} \mu+(1+\kappa \rho) \tau-r_{2} \omega$ and $\mathscr{D}(z ; \rho)=r_{3} \mu+$ $z(1+\kappa \rho) \tau-r_{4} \omega$ are polynomials of degree at most $4 m+1$ in $z$ with a single free parameter $\rho \in \mathbb{C}$. This gives the desired result, completing the proof.

The result of Theorem 7 enables us to discover that $z=0$ (corresponding to fixed point $A$ of $R_{f}$ or root $A$ of $f(z)=$ $\left.[(z-A)(z-B)]^{m}\right)$ and $z=\infty$ (corresponding to fixed point $B$ of $R_{f}$ or root $B$ of $f(z)$ ) are clearly two of their fixed points of the conjugate map $J(z ; \rho)$, regardless of $\rho$-values. Besides, by direct computation, we find that $z=1$ is a strange fixed point [28-30] of $J$ (that is not a root of $f(z)=[(z-A)(z-B)]^{m}$ ) due to the fact that $J(1 ; \rho)=1$, regardless of $\rho$-values.

We now seek further strange fixed points including $z=1$ (corresponding to the original convergence to infinity in view of the fact that $M^{-1}(1)=\infty$ or $\left.M(\infty)=1\right)$. To do so, we first investigate some properties of $J(z ; \rho)=z \cdot(\mathscr{H}(z ; \rho) / \mathscr{D}(z ; \rho))$ stated in the following theorem.

Theorem 8. Let $\mathscr{H}(z ; \rho)$ and $\mathscr{D}(z ; \rho)$ be given by (25). Then the following hold:

(a) The leading highest-order term of $\mathscr{H}(z ; \rho)$ is given by $-\left(8 m^{7 m-4} /(m+2)^{3 m-2}\right)\left[((m+2) / m)^{m-1}+\rho\right] z^{4 m+1}$, provided that $\rho \neq-((m+2) / m)^{m-1}$.

(b) $\mathscr{H}(z ; \rho)$ has a factor $z^{3}$, provided that $\rho \neq-(3 m+$ 4) $\left(5 m^{2}+4\right) \gamma / 2\left(15 m^{3}+20 m^{2}+12 m-8\right) \kappa$.

(c) $\mathscr{H}(1 ; \rho)=\mathscr{D}(1 ; \rho)$, and $\mathscr{H}(1 ; \rho) / \mathscr{D}(1 ; \rho)=1$, provided that $\rho \neq-\left(m^{3}(2 m-\gamma)^{4 m-2}+\tau_{1}-\left(m^{3}+3 m^{2}+\right.\right.$ $\left.2 m+4) \tau_{2}\right) / \kappa\left[\left(m^{3}-4 m-8\right)(2 m-\gamma)^{4 m-2}+\tau_{1}-(m-\right.$ 1) $\left.(m+2)^{2} \tau_{2}\right]$, with $\tau_{1}=4^{2 m-1} m^{4 m-2}(m+2)^{3} \kappa^{2}$ and $\tau_{2}=4^{m} m^{2 m-1}(2 m-\gamma)^{2 m-1} \kappa$.

(d) $J(z ; \rho)$ approaches $\infty$ as $z$ tends to $\infty$, provided that $\rho \neq-((m+2) / m)^{m-1}$.

Proof. After a lengthy computation and careful algebraic treatments with the aid of Mathematica, (a) and (c) follow without difficulty. For the proof of (b), we directly compute the values of $\mathscr{H}(0 ; \rho)=\mathscr{H}^{\prime}(0 ; \rho)=\mathscr{H}^{\prime \prime}(0 ; \rho)=0$ and $\mathscr{H}^{\prime \prime \prime}(0 ; \rho) \neq 0$. The proof of (d) follows from the fact that $J(\infty ; \rho)=\infty$, by using (a) along with a highest-order term of $\mathscr{D}(z ; \rho)$ having degree at most $4 m+1$.

We now will begin with locating the fixed points of the iteration function $J(z ; \rho)$. Let $\phi(z ; \rho)=z-J(z ; \rho)$, whose zeros are the desired fixed points of $J$. The result of Theorem 8 shows that $z=0$ and $z=1$ are the roots of $\phi$. Hence the expression of $\phi(z ; \rho)$ will take the following form:

$$
\phi(z ; \rho)=z(z-1) \cdot \frac{\Psi(z ; \rho)}{T(z ; \rho)},
$$


where $\Psi(z ; \rho)=m^{7} q(z+1)^{4}+m^{2} r_{1} \beta_{1}^{2 m} \beta_{2}^{2 m} \beta^{2}-2 m^{6} q(z+$ $1)^{4}(\gamma-3)+8 q z^{2} \gamma^{4}-2 m^{2 m+1}(z+1)^{2 m} \beta_{1}^{m+1} \beta_{2}^{m+1} \beta \kappa v_{0}-$ $4 m q z \gamma^{3} v_{1}+2 m^{2} q \gamma^{2} v_{2}+m^{5} q(z+1)^{2} v_{3}+2 m^{4} q(z+1)^{2} v_{4}+$ $m^{3} q \gamma v_{5}$ and $T(z ; \rho)=m^{7} q z(z+1)^{4}-2 m^{6} q z(z+1)^{4}(\gamma-$ 3) $+8 q z^{3} \gamma^{4}-2 m^{2 m+1} r_{4}(z+1)^{2 m} \beta_{1}^{m+1} \beta_{2}^{m+1} \beta \kappa-4 m q z^{2} \gamma^{3} \nu_{1}+$ $m^{2}\left(r_{3} \beta_{1}^{2 m} \beta_{2}^{2 m} \beta^{2}+2 q z \gamma^{2} v_{2}\right)+m^{5} q z(z+1)^{2} v_{3}+2 m^{4} q z(z+1)^{2} v_{4}+$ $m^{3} q z \gamma v_{5}$ are polynomials in $z$ with $q=(z+1)^{4 m} m^{4 m} \kappa^{2}(1+$ $\kappa \rho), v_{0}=-4(1+\kappa \rho)+m(2+m(m+3)(1+\kappa \rho)), v_{1}=$ $4(z+1)^{2}-3 z \gamma, v_{2}=4(z+1)^{2}\left(1+4 z+z^{2}\right)-12 z(z+1)^{2} \gamma+$ $3 z^{2} \gamma^{2}, v_{3}=12(z+1)^{2}-12(z+1)^{2} \gamma+\left(1+4 z+z^{2}\right) \gamma^{2}, v_{4}=$ $4(z+1)^{2}-12(z+1)^{2} \gamma+3\left(1+4 z+z^{2}\right) \gamma^{2}-z \gamma^{3}$, and $\nu_{5}=$ $-16(z+1)^{4}+12(z+1)^{2}\left(1+4 z+z^{2}\right) \gamma-12 z(z+1)^{2} \gamma^{2}+z^{2} \gamma^{3}$ and with $\beta_{1}, \beta_{2}, \beta, r_{1}, r_{3}$, and $r_{4}$ given in Theorem 7 .

As a result, $z=0, z=1$, and $z=\infty$ are clearly the fixed points of $J$. Among these fixed points, $z=1$ is a strange fixed point that is not the root $A$ or $B$. Further strange fixed points are possible from the roots of $\Psi(z ; \rho)$. The following theorem describes some properties of $\phi(z ; \rho)$.

Theorem 9. Let $\phi(z ; \rho)$ be given by (26). Then the following hold:

(a) $\Psi(1 / z ; \rho)=\left(1 / z^{4(m+1)}\right) \Psi(z ; \rho)$ for $z \neq 0$, regardless of $\rho$-values.

(b) $\Psi(z ; \rho)$ has double roots at $z=-(m+2) / m$ and $z=$ $-m /(m+2)$; that is, it has a factor $(z+(m+2) / m)^{2}(z+$ $m /(m+2))^{2}$, provided that $\rho \neq-1 / \kappa$.

(c) $T(1 / z ; \rho)=\left(1 / z^{4 m+5}\right) \widetilde{T}(z ; \rho)$ for $z \neq 0$, regardless of $\rho$ values, where $\widetilde{T}(z ; \rho)=\left(T(z ; \rho)-\delta_{0}\right) / z, \delta_{0}=8 m^{2}\left(z^{2}-\right.$ 1) $\beta_{1}^{m+1} \beta_{2}^{m+1} \beta \kappa\left(m^{2 m-1}(z+1)^{2 m}+\beta_{1}^{m-1} \beta_{2}^{m-1} \beta \rho\right)$.

(d) $T(z ; \rho)$ has also double roots at $z=-(m+2) / m$ and $z=-m /(m+2)$; that is, it has a common factor $(z+(m+$ $2) / m)^{2}(z+m /(m+2))^{2}$ as shown in $\Psi(z ; \rho)$, provided that $\rho=-1 / \kappa$.

(e) $\phi(1 / z ; \rho)=-((z-1) / z)$, and $(\Psi(z ; \rho) / \widetilde{T}(z ; \rho))$, for $z \neq 0$, provided that $\rho=-1 / \kappa$.

Proof. Via careful algebraic treatments and symbolic computation with the aid of Mathematica, (a), (c), and (e) follow without difficulty. For the proof of (b), we directly compute the values of $\Psi(-(m+2) / m ; \rho)=\Psi^{\prime}(-(m+2) / m ; \rho)=$ 0 and $\Psi^{\prime \prime}(-(m+2) / m ; \rho)=2^{4(m+1)} m^{3} \gamma \kappa^{2}(m+1)^{2}(1+$ $\kappa \rho) \neq 0$, for $\rho \neq-1 / \kappa$. In view of the relations, $\Psi(z)=$ $z^{4 m+4} \Psi(1 / z), \Psi^{\prime}(z)=z^{4 m+2}\left[4(m+1) z \Psi(1 / z)-\Psi^{\prime}(1 / z)\right]$, and $\Psi^{\prime \prime}(z)=z^{4 m}\left[4\left(4 m^{2}+7 m+3\right) z^{2} \Psi(1 / z)-2(4 m+\right.$ 3) $\left.z \Psi^{\prime}(1 / z)+\Psi^{\prime \prime}(1 / z)\right]$, we also find $\Psi(-m /(m+2) ; \rho)=$ $\Psi^{\prime}(-m /(m+2) ; \rho)=0$ and $\Psi^{\prime \prime}(-m /(m+2) ; \rho)=$ $2^{4(m+1)} m^{3} \gamma \kappa^{6}(m+1)^{2}(1+\kappa \rho) \neq 0$. The proof of (d) follows from the fact that $T(-(m+2) / m ; \rho)=T^{\prime}(-(m+2) / m ; \rho)=$ 0 and $T^{\prime \prime}(-(m+2) / m ; \rho)=\infty$, for any $\rho$. We also find $T(-m /(m+2) ; \rho)=T^{\prime}(-m /(m+2) ; \rho)=0$ and $T^{\prime \prime}(-m /(m+$ 2); $\rho)=-2^{4 m+5} m^{3}(m+1)^{2} \kappa^{2}(1+\kappa \rho) \neq 0$, for $\rho \neq-1 / \kappa$. We also find $T(-m /(m+2) ; \rho)=T^{\prime}(-m /(m+2) ; \rho)=0$ and $T^{\prime \prime}(-m /(m+2) ; \rho)=-2^{4 m+3} m^{3}(m+1)^{2} \gamma^{2} \kappa^{6}(1+\kappa \rho) \neq 0$, for $\rho \neq-1 / \kappa$.
With the use of properties of $\phi(z ; \rho)$, we now consider some strange fixed points along with their stability for selected values of $m=1$ and $m=2$.

To continue our investigation of dynamics behind iterative map (20) applied to a quadratic polynomial raised to the power of $m, f(z)=p(z)=[(z-A)(z-B)]^{m}$, we will describe the fixed points of $J$ in (25) and their stability. In view of the fact that $M(z)$ is a fixed point of $J$ for a fixed point $z$ of $R_{p}$ with $M^{-1}(z)=(z B-A) /(z-1)$, we are interested in the explicit form of $\phi(z ; \rho)=z-J(z ; \rho)$ for $m \in\{1,2\}$ below:

$$
\phi(z ; \rho)= \begin{cases}-\frac{z(z-1) \Psi_{1}(z)}{q_{1}(z)}, & \text { if } m=1, \\ -\frac{z(z-1) \Psi_{2}(z)}{q_{2}(z)}, & \text { if } m=2,\end{cases}
$$

where we conveniently denote

$$
\begin{aligned}
& \Psi_{1}(z)=9(1+\rho)+9 z^{4}(1+\rho)+3 z(11+7 \rho) \\
& \quad+3 z^{3}(11+7 \rho)+z^{2}(54+34 \rho) \\
& q_{1}(z)=9(1+\rho)+12 z(2+\rho)+z^{2}(21+13 \rho) \\
& \Psi_{2}(z)=\left(4(2+\rho)+4 z^{8}(2+\rho)+4 z(17+8 \rho)\right. \\
& \quad+4 z^{7}(17+8 \rho)+3 z^{4}(236+97 \rho) \\
& \quad+z^{2}(256+113 \rho)+z^{6}(256+113 \rho) \\
& \left.\quad+z^{3}(548+230 \rho)+z^{5}(548+230 \rho)\right) \\
& q_{2}(z)=4(2+\rho)+10 z^{5}(12+5 \rho)+4 z(15+7 \rho) \\
& \quad+z^{6}(20+9 \rho)+6 z^{3}(52+21 \rho)+z^{2}(188+81 \rho) \\
& \quad+z^{4}(280+111 \rho) .
\end{aligned}
$$

This enables us to discover that $z=0$ (corresponding to fixed point $a$ of $R_{p}$ or root $A$ of $p(z)$ ) and $z=\infty$ (corresponding to fixed point $B$ of $R_{p}$ or root $B$ of $p(z)$ ) are clearly two of their fixed points regardless of $m$. To find further strange fixed points, we solve equations $\phi(z ; \rho)=0$ in (27) for $z$ with typical values of $m \in\{1,2\}$.

We now investigate further strange fixed points including $z=1$ (corresponding to the original convergence to infinity in view of the fact that $M^{-1}(1)=\infty$ or $\left.M(\infty)=1\right)$. By direct computation, we will describe the roots of $\phi(z ; \rho)=0$ for $m \epsilon$ $\{1,2\}$. To this end, we first check the existence of $\rho$-values for common factors (divisors) of $\Psi_{i}(z)$ and $q_{i}(z)$. Besides, $q_{i}(z)$ will be checked if it has a divisor $(z-1)$ or $z$. The following theorem best describes relevant properties of such existence as well as explicit strange fixed points.

Theorem 10. Let $m=1$ in (27). Then the following hold:

(a) If $\rho=-3$, then $\phi(z ; \rho)=-(z-1) z\left(z^{2}+z+1\right)$ and the strange fixed points $z$ are given by $z=1$ and $z=$ $(-1 \pm \sqrt{3}) / 2$. 
(b) If $\rho=-1$, then $\phi(z ; \rho)=-(z-1) z\left(3 z^{2}+5 z+3\right) /(2 z+3)$ and the strange fixed points are given by $z=1$ and $z=(-5 \pm i \sqrt{11}) / 6$.

(c) If $\rho=-3 / 5$, then $\phi(z ; \rho)=-(z-1) z(z+1)\left(3 z^{2}+11 z+\right.$ $3) /(11 z+3)$ and the strange fixed points are given by $z= \pm 1, z=(-11 \pm \sqrt{85}) / 6$.

(d) If $\rho=-27 / 17$, then $\phi(z ; \rho)=-z\left(15 z^{4}+z^{3}+z+\right.$ $15) /(z+15)$ and the strange fixed points are given by $z=(1 / 60)(-1-\sqrt{1801} \pm i \sqrt{1798-2 \sqrt{1801}})$ and $z=$ $(1 / 60)(-1+\sqrt{1801} \pm i \sqrt{1798+2 \sqrt{1801}})$.

(e) Let $\rho \notin\{-3,-1,-3 / 5,-27 / 17\}$. Then $\Psi_{1}(1 / z)=$ $z^{-4} \Psi_{1}(z)$ holds for $z \neq 0$. Hence, if $z \neq 0$ is a root of $\Psi_{1}(z ; \rho)$, then so is $1 / z$.

Proof. (a)-(c) Suppose that $\Psi_{1}(z)=0$ and $q_{1}(z)=0$ for some values of $z$. Observe that parameter $\rho$ exists in a linear fashion in all coefficients of both polynomials. By eliminating $\rho$ from the two polynomials, we obtain the relation $G(z)=z(1+$ $z)\left(3+2 z+3 z^{2}\right)=0$. Hence, any root of $G$ is a candidate for a common divisor of $\Psi_{1}(z)$ and $q_{1}(z)$. Substituting all the roots of $G$ into $\Psi_{1}(z)=0$ and $q_{1}(z)=0$, we find required relations for $\rho$ and solving them for $\rho$, we find $\rho=-3,-1$. The remaining part of the proof is straightforward. (d) If $z$ is a divisor of $q_{1}(z)$, then $q_{1}(0)=9(1+\rho)=0$ yielding $\rho=-1$, which is already handled in (b). If $(z-1)$ is a divisor of $q_{1}(z)$, then $q_{1}(1)=2(27+17 \rho)=0$, yielding $\rho=-27 / 17$. Then remaining proof is trivial. (e) By direct substitution, we find $\Psi_{1}(1 / z)=z^{-4} \Psi_{1}(z)$ without difficulty. Hence $\Psi_{1}(1 / z)=0$ if and only if $\Psi_{1}(z)=0$ for $z \neq 0$. This completes the proof.

Theorem 11. Let $m=2$ in (27). Then the following hold:

(a) If $\rho=-2$, then $\phi(z ; \rho)=-(z-1) z\left(2 z^{4}+9 z^{3}+15 z^{2}+\right.$ $9 z+2) /\left(z^{3}+7 z^{2}+7 z+2\right)$ and the strange fixed points are given by $z=1, z=-1.80198 \pm 0.880308 i$, and $z=-0.448023 \pm 0.21887 i$.

(b) If $\rho=-4$, then $\phi(z ; \rho)=-(z-1) z(1+z)^{3} /(2 z+1)$ and the strange fixed points are given by $z=1$ and $z=-1$ (triple).

(c) If $\rho=-988 / 409$, then $\phi(z ; \rho)=-z\left(170 z^{8}+\right.$ $951 z^{7}+1735 z^{6}+777 z^{5}-516 z^{4}+777 z^{3}+1735 z^{2}$ $+951 z+170) /\left(178 z^{5}+258 z^{4}-955 z^{3}-1735 z^{2}-\right.$ $951 z-170)$ and the strange fixed points are given by $z=-2.35462,-0.424696,-1.68698 \pm$ $0.790067 i,-0.486146 \pm 0.227678 i, 0.765729 \pm$ $0.643163 i$.

(d) Let $\rho \notin\{-2,-4,-988 / 409\}$. Then $\Psi_{2}(1 / z)=z^{-8} \Psi_{2}(z)$ holds for $z \neq 0$. Hence, if $z \neq 0$ is a root of $\Psi_{2}(z ; \rho)$, then so is $1 / z$.

Proof. The proofs immediately follow from the same argument as used in the proofs of Theorem 10.

As a result of Theorem 9 (a), we find the fixed points of $J(z ; \rho)$, that is, the roots of $\phi(z ; \rho)$ explicitly as stated in the following corollary.
Corollary 12. Let $z \notin\{0,1\}$ be a root of $\phi(z ; \rho)$, that is, a root of $\Psi_{i}(z ; \rho)$ for $1 \leq i \leq 2$ in (27). Suppose that $\Psi(z ; \rho)$ and $q_{i}(z)$ have no common factors for some suitable $\rho$-values. Then the roots of $\phi(z ; \rho)$ for $1 \leq i \leq 2$ are explicitly given by the following:

(a) The four roots of $\Psi_{1}(z ; \rho)$ are explicitly found to be

$$
\begin{aligned}
& z_{1}^{(1)}=-\frac{11+t_{1}+7 \rho+i \sqrt{2} \sqrt{-11 t_{1}+t_{2}-7 t_{1} \rho}}{12(1+\rho)}, \\
& z_{2}^{(1)}=\frac{1}{z_{1}^{(1)}}, \\
& z_{3}^{(1)}=\frac{-11+t_{1}-7 \rho-i \sqrt{2} \sqrt{11 t_{1}+t_{2}+7 t_{1} \rho}}{12(1+\rho)}, \\
& z_{4}^{(1)}=\frac{1}{z_{3}^{(1)}}
\end{aligned}
$$

where $t_{1}=\sqrt{-23-3 \rho(18+5 \rho)}$ and $t_{2}=23+\rho(94+$ $55 \rho)$.

(b) The eight roots of $\Psi_{2}(z ; \rho)$ are explicitly found to be

$$
\begin{aligned}
& z_{1}^{(2)}=\frac{1}{2}\left(-s_{1}-\sqrt{-4+s_{1}^{2}}\right), \\
& z_{2}^{(2)}=\frac{1}{z_{1}^{(2)}}, \\
& z_{3}^{(2)}=\frac{1}{2}\left(-s_{2}-\sqrt{-4+s_{2}^{2}}\right), \\
& z_{4}^{(2)}=\frac{1}{z_{3}^{(2)}}, \\
& z_{5}^{(2)}=\frac{1}{2}\left(-u_{1}-\sqrt{-4+u_{1}^{2}}\right), \\
& z_{6}^{(2)}=\frac{1}{z_{5}^{(2)}}, \\
& z_{7}^{(2)}=\frac{1}{2}\left(-u_{2}-\sqrt{-4+u_{2}^{2}}\right), \\
& z_{8}^{(2)}=\frac{1}{z_{7}^{(2)}},
\end{aligned}
$$

$$
\begin{aligned}
& \text { where } s_{1}=(1 / 2)\left(c_{1}-\sqrt{8+c_{1}^{2}-4 c_{2}}\right), s_{2}=(1 / 2)\left(c_{1}+\right. \\
& \left.\sqrt{8+c_{1}^{2}-4 c_{2}}\right), u_{1}=(1 / 2)\left(d_{1}-\sqrt{8+d_{1}^{2}-4 d_{2}}\right), u_{2}= \\
& (1 / 2)\left(d_{1}+\sqrt{8+d_{1}^{2}-4 d_{2}}\right), c_{1}=(17+8 \rho- \\
& \left.\sqrt{-7-6 \rho-C^{2}}\right) / 2(2+\rho), c_{2}=3(9+4 \rho- \\
& \left.\sqrt{-7-6 \rho-\rho^{2}}\right) / 2(2+\rho), d_{1}=(17+8 \rho+ \\
& \left.\sqrt{-7-6 \rho-\rho^{2}}\right) / 2(2+\rho), \text { and } d_{2}=3(9+4 \rho+ \\
& \left.\sqrt{-7-6 \rho-\rho^{2}}\right) / 2(2+\rho) .
\end{aligned}
$$


Proof. Since $z$ is a root of $\Psi_{i}(z ; \rho)$ for $1 \leq i \leq 2$, so is $1 / z$ from the result of Theorem 9 (a). For the proof of (a), $\Psi_{1}(z ; \rho)$ can be written as a product of two factors:

$$
\Psi_{1}(z ; \rho)=\left(z^{2}+c z+1\right)\left(z^{2}+d z+1\right) .
$$

By expanding the right side of the above equation and comparing the coefficients of the first- and second-order terms, we find two relations:

$$
\begin{aligned}
c+d & =\frac{11+7 \rho}{3(1+\rho)}, \\
2+c d & =\frac{54+34 \rho}{9(1+\rho)},
\end{aligned}
$$

which gives the desired values of $c$ and $d$. Then the four roots can be found explicitly from $\left(z^{2}+c z+1\right)=0$ or $\left(z^{2}+d z+1\right)=$ 0 . Similarly for the proof of (b), $\Psi_{2}(z ; \rho)$ can be written as a product of two factors each of which is further decomposed into two factors:

$$
\begin{aligned}
& \Psi_{2}(z ; \rho)=\left(1+c_{1} z+c_{2} z^{2}+c_{1} z^{3}+z^{4}\right) \\
& \cdot\left(1+d_{1} z+d_{2} z^{2}+d_{1} z^{3}+z^{4}\right)=\left(z^{2}+s_{1} z+1\right) \\
& \cdot\left(z^{2}+s_{2} z+1\right)\left(z^{2}+u_{1} z+1\right)\left(z^{2}+u_{2} z+1\right) .
\end{aligned}
$$

By the same argument as used in the proof of (a), the desired result follows. This completes the proof.

We are now ready to determine the stability of the fixed points. To do so, it is necessary to compute the derivative of $J$ from (24):

$$
J^{\prime}(z ; \rho)= \begin{cases}\frac{4 z^{3} Q_{1}(z)}{w_{1}(z)^{2}}, & \text { if } m=1, \\ \frac{2 z^{3} Q_{2}(z)}{w_{2}(z)^{2}}, & \text { if } m=2,\end{cases}
$$

where

$$
\begin{aligned}
Q_{1}(z)= & 36 z\left(18+23 \rho+7 \rho^{2}\right) \\
& +36 z^{3}\left(18+23 \rho+7 \rho^{2}\right) \\
& +9\left(21+34 \rho+13 \rho^{2}\right) \\
& +9 z^{4}\left(21+34 \rho+13 \rho^{2}\right) \\
& +2 z^{2}\left(459+546 \rho+175 \rho^{2}\right) \\
w_{1}(z)= & 9(1+\rho)+12 z(2+\rho)+z^{2}(21+13 \rho), \\
Q_{2}(z)= & \left(40+38 \rho+9 \rho^{2}\right)+8 z^{12}\left(40+38 \rho+9 \rho^{2}\right) \\
& +z\left(4200+3850 \rho+878 \rho^{2}\right) \\
& +z^{11}\left(4200+3850 \rho+878 \rho^{2}\right)
\end{aligned}
$$

$$
\begin{aligned}
& +z^{2}\left(24880+22056 \rho+4861 \rho^{2}\right) \\
& +z^{10}\left(24880+22056 \rho+4861 \rho^{2}\right) \\
& +2 z^{3}\left(43848+37745 \rho+8088 \rho^{2}\right) \\
& +2 z^{9}\left(43848+37745 \rho+8088 \rho^{2}\right) \\
& +6 z^{5}\left(55820+46235 \rho+9581 \rho^{2}\right) \\
& +6 z^{7}\left(55820+46235 \rho+9581 \rho^{2}\right) \\
& +2 z^{4}\left(102448+86152 \rho+18081 \rho^{2}\right) \\
& +2 z^{8}\left(102448+86152 \rho+18081 \rho^{2}\right) \\
& +z^{6}\left(393440+324176 \rho+66891 \rho^{2}\right) \\
w_{2}(z)= & (2+\rho)+10 z^{5}(12+5 \rho)+4 z(15+7 \rho) \\
& +z^{6}(20+9 \rho)+6 z^{3}(52+21 \rho) \\
& +z^{2}(188+81 \rho)+z^{4}(280+111 \rho)
\end{aligned}
$$

We first check the existence of $\rho$-values for common factors (divisors) of $Q_{i}(z)$ and $w_{i}(z)$. Besides, $w_{i}(z)$ will be checked if it has divisors $z, z^{2}, z^{3}$. The following theorem best describes relevant properties of such existence as well as explicit strange fixed points.

Theorem 13. Let $m=1$ in (34). Then the following hold:

(a) If $\rho=-3$, then $J^{\prime}(z ; \rho)=4 z^{3}$.

(b) If $\rho=-1$, then $J^{\prime}(z ; \rho)=2 z^{2}\left(9 z^{2}+22 z+9\right) /(2 z+3)^{2}$.

(c) If $\rho=-3 / 5$, then $J^{\prime}(z ; \rho)=12 z^{3}\left(11 z^{2}+34 z+\right.$ $11) /(11 z+3)^{2}$.

(d) If $\rho=-1 / 3$, then $J^{\prime}(z ; \rho)=4 z^{3}(3 z+5)\left(15 z^{2}+26 z+\right.$ $15) /(5 z+3)^{3}$.

(e) If $\rho=-5 / 3$, then $J^{\prime}(z ; \rho)=4 z^{3}(3 z-1)\left(3 z^{2}-14 z+\right.$ 3) $/(z-3)^{3}$.

(f) If $\rho=-27 / 17$, then $J^{\prime}(z ; \rho)=-12 z^{3}\left(5+94 z+5 z^{2}\right) /(z+$ $15)^{2}$.

(g) Let $\rho \notin\{-3,-1,-3 / 5,-1 / 3,-5 / 3,-27 / 17\}$. Let $z$ be a fixed point of $J(z ; \rho)$ satisfying $\phi(z ; \rho)=0$. Then $J^{\prime}(z ; \rho)=J^{\prime}(1 / z ; \rho)$ holds for $z \neq 0$.

Proof. The proofs of (a)-(f) immediately follow from the same argument as used in the proofs of Theorem 10. Eliminating $\rho$ from the two polynomials $Q_{i}(z)$ and $w_{i}(z)$ plays a key role in obtaining the relation $G(z)=(-3+z)(-1+z) z(1+$ $z)(3+5 z)\left(3+2 z+3 z^{2}\right)=0$, whose roots enable us to deduce some desired $\rho$-values. Additional requirement that $z, z^{2}, z^{3}$ are candidates for common divisors of $Q_{i}(z)$ and $w_{i}(z)$ gives only $\rho=-1$. For the proof of $(\mathrm{g})$, via direct computation with the aid of Mathematica symbolic capability, we find $J^{\prime}(z ; \rho)-$ $J^{\prime}(1 / z ; \rho)=-\left(4 Q_{1}(z)(z+1) \Delta_{1} / q_{1}(z) q_{1}(1 / z)^{2} z^{8}\right) \phi(z ; \rho)=0$, 
TABLE 2: Stability check from $\left|J^{\prime}(\zeta ; \rho)\right|$ of strange fixed points $\zeta$ for special $\rho$-values with $1 \leq m \leq 2$.

\begin{tabular}{|c|c|c|c|c|c|c|c|}
\hline$m$ & $\rho$ & & & $\begin{array}{c}\zeta \\
\left|J^{\prime}(\zeta ; \rho)\right|: \mathbf{t}^{*}\end{array}$ & & & Number of $\zeta$ \\
\hline \multirow{12}{*}{1} & \multirow{2}{*}{$-3 / 5$} & -1 & 1 & $(-11 \pm \sqrt{85}) / 6$ & & & \multirow{2}{*}{4} \\
\hline & & $9 / 4: \mathbf{r}$ & $24 / 7: \mathbf{r}$ & 76/9: $\mathbf{r}$ & & & \\
\hline & \multirow{2}{*}{-3} & 1 & $\pm(1+\sqrt{3}) / 2$ & & & & \multirow{2}{*}{3} \\
\hline & & $4: \mathbf{r}$ & $4: \mathbf{r}$ & & & & \\
\hline & \multirow[t]{2}{*}{-1} & 1 & $(-5 \pm i \sqrt{11}) / 6$ & & & & \multirow[t]{2}{*}{3} \\
\hline & & $16 / 5: \mathbf{r}$ & 14/3: r & & & & \\
\hline & \multirow[t]{2}{*}{$-1 / 3$} & 1 & $\begin{array}{c}-1.70469 \pm \\
0.885458 i\end{array}$ & $-0.461974 \pm 0.239960 i$ & & & \multirow[t]{2}{*}{5} \\
\hline & & $7 / 2: \mathbf{r}$ & 4.76095: r & 4.76095: $\mathbf{r}$ & & & \\
\hline & \multirow[t]{2}{*}{$-5 / 3$} & 1 & $\begin{array}{c}-0.712486 \pm \\
0.701686 i\end{array}$ & $0.545819 \pm 0.837902 i$ & & & \multirow[t]{2}{*}{5} \\
\hline & & $8: \mathbf{r}$ & 5.12085: $\mathbf{r}$ & 6.37915: $\mathbf{r}$ & & & \\
\hline & \multirow[t]{2}{*}{$-27 / 17$} & $\begin{array}{c}-0.723969 \pm \\
0.689831 i\end{array}$ & $\begin{array}{c}0.690636 \pm \\
0.723202 i\end{array}$ & & & & \multirow[t]{2}{*}{4} \\
\hline & & 5.09653: $\mathbf{r}$ & 4.90792: $\mathbf{r}$ & & & & \\
\hline \multirow{6}{*}{2} & -2 & 1 & $\begin{array}{c}-1.80197 \pm \\
0.880307 i\end{array}$ & $-0.448023 \pm 0.218869 i$ & & & \multirow[t]{2}{*}{5} \\
\hline & & $54 / 17: \mathbf{r}$ & 6.49038: $\mathbf{r}$ & 6.49038: $\mathbf{r}$ & & & \\
\hline & \multirow{2}{*}{-4} & -1 (triple) & 1 & & & & \multirow{2}{*}{4} \\
\hline & & $1: \mathbf{p}$ & $11 / 3: \mathbf{r}$ & & & & \\
\hline & \multirow[t]{2}{*}{$-988 / 409$} & -2.35462 & $\begin{array}{c}-1.68698 \pm \\
0.790067 i\end{array}$ & $0.765729 \pm 0.643163 i$ & $-0.486146 \pm 0.227678 i$ & -0.424696 & \multirow[t]{2}{*}{8} \\
\hline & & 834.411: $\mathbf{r}$ & 6.04594: $\mathbf{r}$ & 4.98848: $\mathbf{r}$ & 6.04594: $\mathbf{r}$ & 834.411: $\mathbf{r}$ & \\
\hline
\end{tabular}

${ }^{*}\left|J^{\prime}(\zeta ; \rho)\right|: \mathbf{t}$ implies that $\zeta$ is attractive, parabolic, and repulsive, if $\mathbf{t}=\mathbf{a}\left(\left|J^{\prime}\right|<1\right), \mathbf{t}=\mathbf{p}\left(\left|J^{\prime}\right|=1\right)$, and $\mathbf{t}=\mathbf{r}\left(\left|J^{\prime}\right|>1\right)$, respectively.

where $\Delta_{1}=9(1+\rho)+3 z(5+\rho)+2 z^{2}(3+5 \rho)+3 z^{3}(5+\rho)+$ $9 z^{4}(1+\rho)$. This completes the proof.

Theorem 14. Let $m=2$ in (34). Then the following hold:

(a) If $\rho=-2$, then $J^{\prime}(z ; \rho)=2 z^{2}\left(3+35 z+112 z^{2}+159 z^{3}+\right.$ $\left.112 z^{4}+35 z^{5}+3 z^{6}\right) /\left(2+7 z+7 z^{2}+z^{3}\right)^{2}$.

(b) If $\rho=-4$, then $J^{\prime}(z ; \rho)=z^{3}\left(8+17 z+8 z^{2}\right) /(1+2 z)^{2}$.

(c) If $\rho=-988 / 409$, then $J^{\prime}(z ; \rho)=z^{3} \sigma_{1} /(-170-951 z-$ $\left.1735 z^{2}-955 z^{3}+258 z^{4}+178 z^{5}\right)^{2}$, where $\sigma_{1}=121040+$ $727134 z+624992 z^{2}-5092795 z^{3}-17328190 z^{4}-$ $24412987 z^{5}-17328190 z^{6}-5092795 z^{7}+624992 z^{8}+$ $727134 z^{9}+121040 z^{10}$.

(d) Let $\rho \notin\{-2,-4,-988 / 409\}$. Let $z$ be a fixed point of $J(z ; \rho)$ satisfying $\phi(z ; \rho)=0$. Then $J^{\prime}(z ; \rho)=J^{\prime}(1 / z$; $\rho)$ holds for $z \neq 0$.

Proof. The proofs of (a)-(c) immediately follow from the same argument as used in the proofs of Theorem 13. For the proof of $(d)$, via direct computation with the aid of Mathematica symbolic capability, we find $J^{\prime}(z ; \rho)-J^{\prime}(1 / z ; \rho)=$ $-\left(2 Q_{2}(z)(z+1) \Delta_{2} / q_{2}(z) q_{2}(1 / z)^{2} z^{16}\right) \phi(z ; \rho)=0$, where $\Delta_{2}=$ $4(2+\rho)+4 z^{8}(2+\rho)+4 z(13+6 \rho)+4 z^{7}(13+6 \rho)+2 z^{3}(98+39 \rho)+$
$2 z^{5}(98+39 \rho)+z^{2}(136+57 \rho)+z^{6}(136+57 \rho)+z^{4}(204+83 \rho)$. This completes the proof.

Table 2 summarizes the stability results for the strange fixed points $\zeta$ of $J$ for special $\rho$-values with $m \in\{1,2\}$.

We are ready to discuss the stability of the fixed points described in Theorems 10 and 11 in terms of parameter $\rho$.

Theorem 15. Let $m=1$ and $\rho \notin\{-3,-1,-3 / 5,-1 / 3,-5 / 3$, $-27 / 17\}$. Then the following hold:

(a) The strange fixed point $z=1$ becomes an attractor, a parabolic (indifferent, neutral) point, and a repulser, respectively, when $|(3+2 \rho) /(27+17 \rho)|<1 / 32, \mid(3+$ $2 \rho) /(27+17 \rho) \mid=1 / 32$, and $|(3+2 \rho) /(27+17 \rho)|>$ $1 / 32$.

(b) The strange fixed point $z=1$ is a superattractor if $\rho=$ $-3 / 2$.

Proof. (a) From the case of $m=1$ in (34), we find $J^{\prime}(1$; $\rho)=32((3+2 \rho) /(27+17 \rho))$. Solving $\left|J^{\prime}(1 ; \rho)\right|=32 \mid(3+$ $2 \rho) /(27+17 \rho) \mid=1$ for $\rho$, we obtain an ellipse $(x+$ $5685 / 3807)^{2} /(32 / 1269)^{2}+y^{2} /\left(32 /(3 \sqrt{206095})^{2}\right)=1$ in the cross-sectional $\rho$-parameter plane for $z=1$ to be a parabolic point, where $x=\operatorname{Re}(\rho), y=\operatorname{Im}(\rho)$. (b) Solving $\left|J^{\prime}(1 ; \rho)\right|=0$ easily yields $\rho=-3 / 2$. 

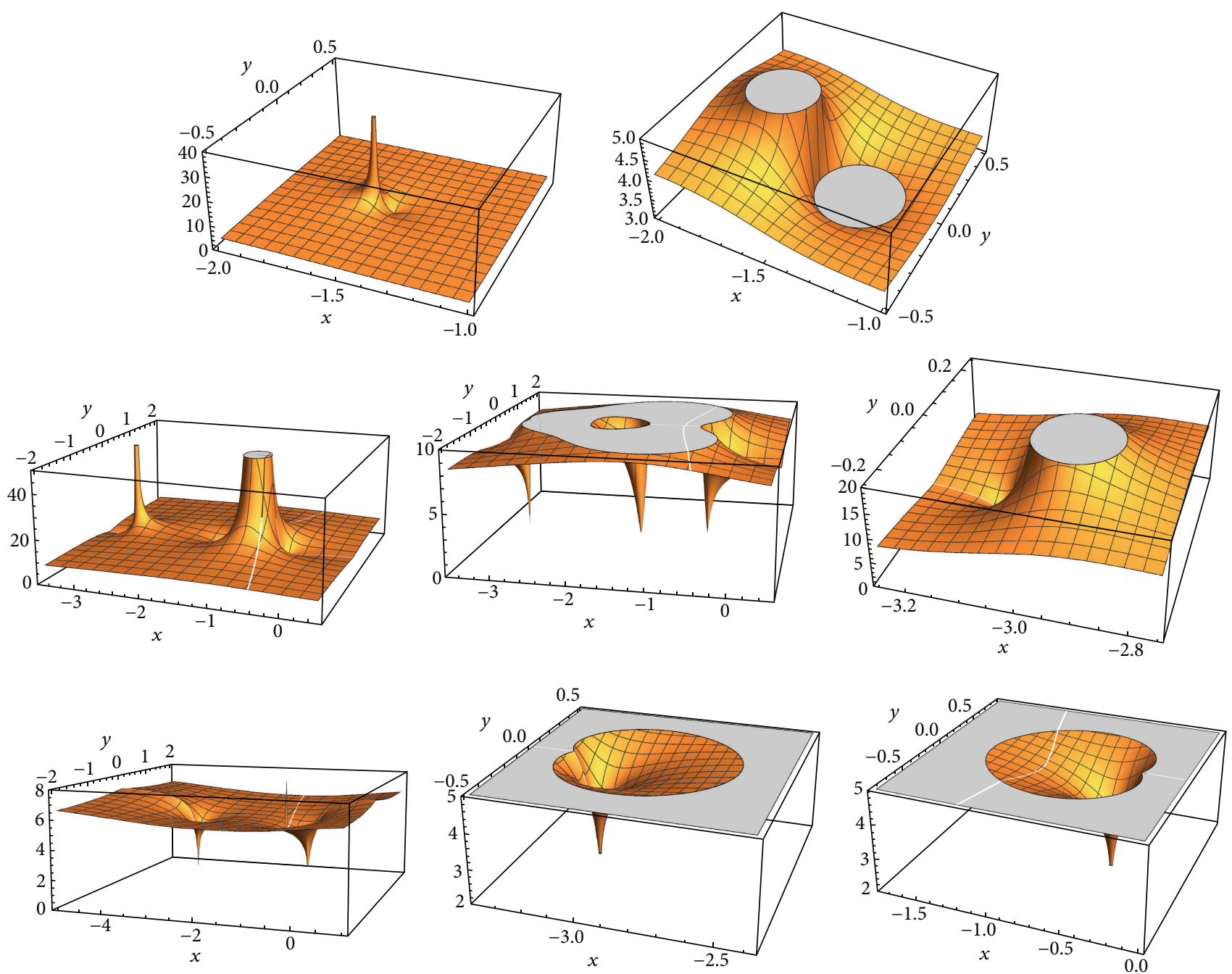

FIgURE 1: Stability surfaces of the strange fixed points $J(z ; \rho)$ for $m=1$.

Theorem 16. Let $m=2$ and $\rho \notin\{-2,-4,-988 / 409\}$. Then the following hold:

(a) The strange fixed point $z=1$ is a parabolic (neutral, indifferent) point, respectively, when $\mid(64+27 \rho) /(988+$ $409 \rho)|<1 / 54|,(64+27 \rho) /(988+409 \rho) \mid=1 / 54$, and $|(64+27 \rho) /(988+409 \rho)|>1 / 54$.

(b) The strange fixed point $z=1$ is a superattractor if $\rho=$ $-64 / 27$.

Proof. From the case of $m=2$ in (34), we find $J^{\prime}(1$; $\rho)=54((64+27 \rho) /(988+409 \rho))$. Solving $\left|J^{\prime}(1 ; \rho)\right|=$ $54|(64+27 \rho) /(988+409 \rho)|=1$ for $\rho$, we obtain an ellipse $(x+4634756 / 1958483)^{2} /(27000 / 1958483)^{2}+$ $y^{2} /(5400 \sqrt{5 / 898177930147})^{2}=1$ in the cross-sectional $\rho$ parameter plane for $z=1$ to be a parabolic point, where $x=\operatorname{Re}(\rho), y=\operatorname{Im}(\rho)$. (b) Solving $\left|J^{\prime}(1 ; \rho)\right|=0$ easily yields $\rho=-64 / 27$.

We now proceed to discuss the stability of the strange fixed points $\zeta$ for conjugate map $J(z ; \rho)$ with $m \in\{1,2\}$ using $J^{\prime}(\zeta ; \rho)$. As a consequence of Theorems $13(\mathrm{~g})$ and $14(\mathrm{~d})$ together with Corollary 12 , the stability can be stated at most five strange fixed points including $z=1$. Then the stability of these fixed points can be best described by illustrative conical surfaces shown in Figures 1-2. The top row of each figure refers to a stability surface for strange fixed point $z=1$. The stability surfaces for the remaining fixed points $z_{i}(1 \leq i \leq 8)$ are displayed in order from top to bottom and from left to right in each case of $m=1$ and $m=2$. The underlying theory is clearly verified via cross-sectional views of the stability surfaces with $\rho$-parameter domains.

\section{Extraneous Fixed Points}

In this section, we will consider different complex dynamics behind the extraneous fixed points to be defined now. The fixed points of $R_{f}$ are zeros of $f(x)$ under consideration. The iteration function $R_{f}$, however, might possess other fixed points that are not zeros of $f$. Such fixed points different from zeros of $f$ are called the extraneous fixed points [31, 32] of the iteration function $R_{f}$. Extraneous fixed points may 

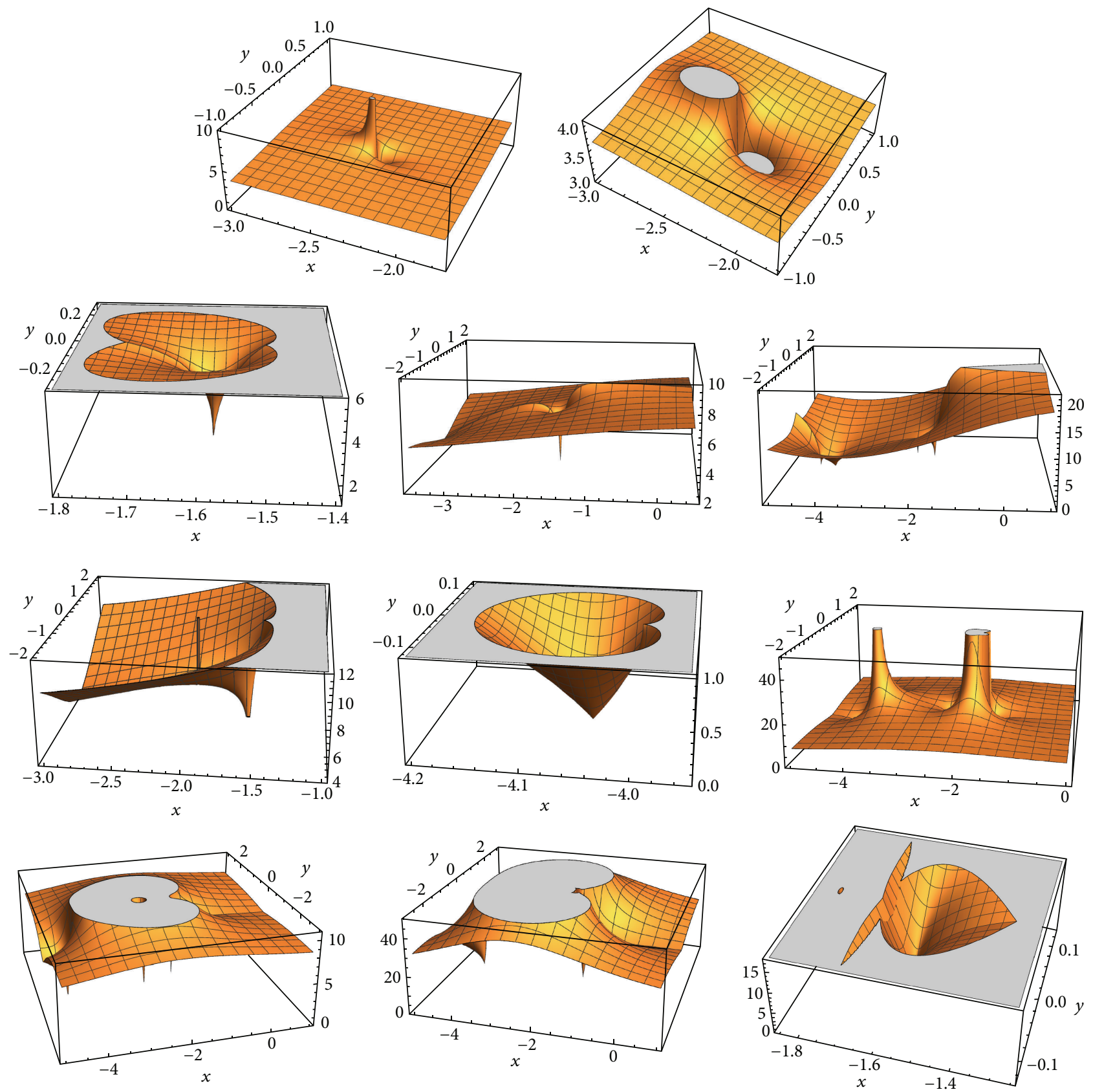

FIGURE 2: Stability surfaces of the strange fixed points $J(z ; \rho)$ for $m=2$.

form attractive, indifferent, repulsive cycles or periodic orbits to display chaotic dynamics behind the basin of attraction under investigation. The existence of such extraneous fixed points would affect the global iteration dynamics, which was demonstrated via König functions by Vrscay and Gilbert [32]. Particularly the presence of attractive cycles induced by the extraneous fixed points of $R_{f}$ may alter the basin of attractions due to the trapped sequence $\left\{x_{n}\right\}$. Even in the case of repulsive or indifferent fixed points, an initial value $x_{0}$ chosen near a desired root may converge to another unwanted remote root. Indeed, these aspects of the Schröder functions $[32,33]$ were observed in an application to the family of functions $\left\{f_{k}(x)=x^{k}-1, k \geq 2\right\}$ for simpleroot finders. By taking a particular member of the family into account for multiple-root finders, we are further interested in the dynamics applied to prototype quadratic polynomial $\left(z^{2}-1\right)$ raised to the power $m$, being the multiplicity of zero $\alpha$ under consideration. Such dynamical aspects motivate our investigation of the extraneous fixed points that may affect the basins of attraction for the proposed methods (5).

The structure of $H_{f}\left(x_{n}\right)$ in (20) clearly characterizes a variety of iterative methods. The zero $\alpha$ of $f(x)$ is obviously a fixed point of $R_{f}$. The points $\xi \neq \alpha$ for which $H_{f}(\xi)=0$ are extraneous fixed points of $R_{f}$. 
Let $H(z)$ represent $H_{f}(z)$ when $f(z)$ is a finite-order rational function of $z$. Then it would be of great interest for us to investigate the complex dynamics of the rational iterative map $R_{p}$ of the form [26]

$$
z_{n+1}=R_{p}\left(z_{n}\right)=z_{n}-\frac{p\left(z_{n}\right)}{p^{\prime}\left(z_{n}\right)} H_{p}\left(z_{n}\right),
$$

in connection with the basins of attraction for a variety of polynomials $p\left(z_{n}\right)$. Clearly, $R_{p}(z)$ represents classical Newton's method with weight function $H_{p}(z)$ and may possess its fixed points as zeros of $p(z)$ or extraneous fixed points associated with $H(z)$.

We now turn to complex dynamics [28-30] behind the basins of attraction of iterative map (36) applied to a prototype quadratic polynomial raised to the power of $m$. We are interested also in the investigation of unified dynamics associated with these extraneous fixed points. To this end, we apply a simple quadratic polynomial raised to the power of multiplicity $m$, that is, $f(z)=\left(z^{2}-1\right)^{m}$ to $H_{f}\left(x_{n}\right)$, simple-root cases of which were introduced by Cayley [34] and Vrscay and Gilbert [32] in dynamical studies of the Schröder and König functions for a family of functions $f_{k}(z)=z^{k}-1, k \in \mathbb{N}$, to minimize perturbations of the Julia set boundaries.

Hence in this section we will discuss the complex dynamics of (36) associated with its extraneous fixed points. To this end, we first write $H(z)$ associated with $H_{f}$ applied to $f(z)=\left(z^{2}-1\right)^{m}$ in the form of

$$
H(z)=\omega+\frac{F(\zeta)}{D(\zeta)}
$$

where $\zeta=z^{2}$ and $\omega=a+c+\gamma(1-d \lambda)$ with $\gamma=2 m /(m+2)$ is a constant independent of $z$;

$$
\begin{aligned}
F(\zeta)= & (b+d)(2 m)^{4 m-2} \zeta^{2 m} \\
& +\rho(b+d)(2 m)^{2 m-1} \delta_{1}^{m-1} \delta_{2} \zeta^{m}
\end{aligned}
$$

$$
\begin{gathered}
-\lambda \gamma(c-d \rho) \delta_{1}^{2 m-2} \delta_{2}^{2} \\
D(\zeta)=\delta_{1}^{m-1} \delta_{2}\left[(2 m)^{2 m-1} \zeta^{m}+\delta_{1}^{m-1} \delta_{2} \rho\right]
\end{gathered}
$$

are polynomials having no common factors with

$$
\begin{aligned}
& \delta_{1}=(2 m-\gamma)^{2} \zeta-\gamma^{2}, \\
& \delta_{2}=(2 m-\gamma) \zeta+\gamma .
\end{aligned}
$$

Hence, the roots of $F(\zeta)+\omega \cdot D(\zeta)=0$ may indeed express the desired extraneous fixed points of $H$, provided that $D(\zeta) \neq 0$. In this paper, we limit ourselves to considering a simple form of $H(z)$ by selecting $\lambda=-m(m+2) / \tau \gamma \kappa, \rho=-m(m+2) / \tau \kappa$, and $d=-m^{2}(m+4)^{3} \kappa /(m+2)^{2} \tau$ with $\tau=m^{2}+2 m-4$ and $\kappa=(m /(m+2))^{m}$ so that we have $a=c=0$ and $b=$ $-m\left(m^{3}+6 m^{2}+8 m-8\right) \kappa / 2(m+2)^{2}$. Consequently, $F(\zeta)$ and $D(\zeta)$ reduce to the following:

$$
\begin{aligned}
& F(\zeta)=\frac{m^{4}}{\tau}\left[-\frac{(m+4)^{3} \delta_{1}^{2 m-2} \delta_{2}^{2}}{\kappa \tau^{2}}\right. \\
& +\frac{2^{2 m-2} m^{2 m-3}(m+2)^{4} \delta_{1}^{m-1} \delta_{2} \zeta^{m}}{\tau} \\
& \left.-2^{4 m-3} m^{4 m-5}(m+2)^{3} \zeta^{2 m} \kappa\right] \\
& D(\zeta)=\delta_{1}^{m-1} \delta_{2}\left[(2 m)^{2 m-1} \zeta^{m}-\frac{m(m+2)}{\tau \kappa} \delta_{1}^{m-1} \delta_{2}\right] .
\end{aligned}
$$

In order to compare the dynamics behavior of (36) behind the extraneous fixed points, let us now investigate the corresponding $H(z)$ of the existing three optimal methods, Kan, Sol, and Li, introduced in Section 1. By similarly following the development procedure of $H(z)$ as shown in (37), we find with $\zeta=z^{2}$

$$
H(z)= \begin{cases}\frac{m\left[-(3 m-2) \delta_{1}^{2 m-2} \delta_{2}^{2}+2^{2 m-1} m^{2 m-2}(2 m+7)(3 m-2) \delta_{1}^{m-1} \delta_{2} \zeta^{m} \kappa-2^{4 m-2} m^{4 m-4} \eta_{1} \zeta^{2 m} \kappa^{2}\right]}{2\left[\delta_{1}^{2 m-2} \delta_{2}^{2}-2^{2 m-1} m^{2 m-2}(2 m+7) \delta_{1}^{m-1} \delta_{2} \zeta^{m} \kappa+2^{4 m-2}(m-1)(m+8) m^{4 m-4} \zeta^{2 m} \kappa^{2}\right]} & \text { for Kan, } \\ \frac{-2^{-2 m}(m-2) m^{2-2 m} \delta_{1}^{2 m-2} \delta_{2}^{2}+(m-2)(m+2) \delta_{1}^{m-1} \delta_{2} \zeta^{m} \kappa-2^{2 m-2} m^{2 m-3} \eta_{2} \zeta^{2 m} \kappa^{2}}{2 \kappa \zeta^{m}\left[\delta_{1}^{m-1} \delta_{2}-2^{2 m-1} m^{2 m-3}\left(m^{2}+2 m-4\right) \zeta^{m} \kappa\right]} & \text { for Sol, } \\ \frac{m\left[-(m-2) \delta_{1}^{m-1} \delta_{2}+2^{2 m-1} m^{2 m} \zeta^{m} \kappa\right]}{2\left[\delta_{1}^{m-1} \delta_{2}-(2 m)^{2 m-1} \zeta^{m} \kappa\right]} & \text { for Li, }\end{cases}
$$

where $\eta_{1}=3 m^{3}+19 m^{2}+16 m+16$ and $\eta_{2}=m^{4}+2 m^{3}-$ $4 m^{2}-8 m-16$.

Since $H(z)$ in (40) or (41) defines a high-order rational function as the multiplicity $m$ increases, it is convenient to study the typical cases of $m \in\{2,3,4,5\}$ for locating the corresponding extraneous fixed points $\xi$ by solving $H(\xi)=$ 0 for $\xi=\zeta^{1 / 2}$. In fact, Table 3 lists the extraneous fixed points $\xi$ and their stability from the value of $\left|R_{p}^{\prime}(\xi)\right|$ applied to a prototype polynomial $p(z)=\left(z^{2}-1\right)^{m}$, respectively, for values of $2 \leq m \leq 5$. As can be seen in the table, all extraneous fixed points of the listed methods are found to be repulsive. Observe that methods Sol and $\mathrm{Li}$ do not possess the extraneous fixed points when $m=2$. In addition, critical points of the proposed methods applied to a polynomial 
TABLE 3: Extraneous fixed points $\xi=\zeta^{1 / 2}$ with stability check for selected cases with $2 \leq m \leq 5$.

\begin{tabular}{|c|c|c|c|c|c|c|}
\hline \multirow[t]{2}{*}{ Method } & \multirow[t]{2}{*}{$m$} & \multirow{2}{*}{$\xi$} & \multirow{2}{*}{ Number of $\xi$ points } & \multicolumn{3}{|c|}{$\left|R_{p}^{\prime}(\xi)\right|$} \\
\hline & & & & $<1$ & 1 & $>1$ \\
\hline \multirow{4}{*}{$Y k$} & 2 & $\pm 0.243414 \pm 0.415875 i, \pm 0.326691 \pm 0.0719699 i$ & 8 & 0 & 0 & 8 \\
\hline & 3 & $\pm 0.248244 \pm 0.321071 i, \pm 0.201519 \pm 0.00811083 i, \pm 0.287319 \pm 0.157274 i$ & 12 & 0 & 0 & 12 \\
\hline & 4 & $\begin{array}{l} \pm 0.180609 \pm 0.209976 i, \pm 0.154424 \pm 0.0318211 i, \pm 0.165971 \pm \\
0.0281012 i, \pm 0.341376 \pm 0.250384 i\end{array}$ & 16 & 0 & 0 & 16 \\
\hline & 5 & $\begin{array}{l}( \pm 0.134189 \pm 0.189108 i, \pm 0.129334 \pm 0.0459184 i, \pm 0.12138, \pm 0.132268, \pm 0.144672 \pm \\
0.0387865 i, \pm 0.380633 \pm 0.254445 i)\end{array}$ & 20 & 0 & 0 & 20 \\
\hline \multirow{4}{*}{ Kan } & 2 & $\pm 0.372622 \pm 0.342462 i, \pm 0.364216 \pm 0.112918 i$ & 8 & 0 & 0 & 8 \\
\hline & 3 & $\pm 0.370756 \pm 0.347075 i, \pm 0.206707 \pm 0.00369212 i, \pm 0.361833 \pm 0.157914 i$ & 12 & 0 & 0 & 12 \\
\hline & 4 & $\begin{array}{l} \pm 0.36607 \pm 0.331711 i, \pm 0.166419 \pm 0.0262382 i, \pm 0.167623 \pm 0.029141 i, \pm 0.362159 \pm \\
0.184595 i\end{array}$ & 16 & 0 & 0 & 16 \\
\hline & 5 & $\begin{array}{l}( \pm 0.132208 \pm 0.000478097 i, \pm 0.144187 \pm 0.038215 i, \pm 0.145007 \pm \\
0.039469 i, \pm 0.362075 \pm 0.308059 i, \pm 0.361766 \pm 0.208745 i)\end{array}$ & 20 & 0 & 0 & 20 \\
\hline \multirow{4}{*}{ Sol } & 2 & - & - & - & - & - \\
\hline & 3 & $\pm 0.679565 i, \pm 0.205392, \pm 0.218593, \pm 0.37247 \pm 0.238124 i, \pm 0.329022$ & 12 & 0 & 0 & 12 \\
\hline & 4 & $\begin{array}{l} \pm 0.778239 i, \pm 0.167222 \pm 0.0275903 i, \pm 0.171541 \pm 0.0256375 i, \pm 0.402522 \pm \\
0.246961 i, \pm 0.351918\end{array}$ & 16 & 0 & 0 & 16 \\
\hline & 5 & $\begin{array}{l}( \pm 0.831769 i, \pm 0.132545, \pm 0.134193, \pm 0.145034 \pm 0.0385469 i, \pm 0.147144 \pm \\
0.0370658 i, \pm 0.419276 \pm 0.24999 i, \pm 0.367148)\end{array}$ & 20 & 0 & 0 & 20 \\
\hline \multirow{4}{*}{$\mathrm{Li}$} & 2 & - & - & - & - & - \\
\hline & 3 & $\pm 0.202285, \pm 0.316011 \pm 0.237459 i$ & 6 & 0 & 0 & 6 \\
\hline & 4 & $\pm 0.166502 \pm 0.0278872 i, \pm 0.363341 \pm 0.25042 i$ & 8 & 0 & 0 & 8 \\
\hline & 5 & $\pm 0.132338, \pm 0.144764 \pm 0.0387259 i, \pm 0.389278 \pm 0.253836 i$ & 10 & 0 & 0 & 10 \\
\hline
\end{tabular}

TABLE 4: Critical points $w$ of $R_{f}$ applied to a polynomial $f(z)=\left(z^{2}-1\right)^{m}$ for selected values of $2 \leq m \leq 5$.

\begin{tabular}{llr}
\hline$m$ & $w$ & Number of $w$ points \\
\hline 2 & $\pm 0.61585 i, \pm 0.300682 i, \pm 0.22275, \pm 0.323127 \pm 0.165034 i, \pm 0.340261, \pm 1.0, \pm 1.0, \pm 1.0$ & 18 \\
\hline 3 & $( \pm 0.555234 i, \pm 0.250157 i, \pm 0.158737 \pm 0.0554075 i, \pm 0.274347 \pm$ & 24 \\
& $0.210868 i, \pm 0.198344, \pm 0.227118, \pm 0.291901, \pm 1.0, \pm 1.0, \pm 1.0)$ & 30 \\
\hline 4 & $( \pm 0.509498 i, \pm 0.214305 i, \pm 0.245897 \pm 0.225504 i, \pm 0.125963 \pm 0.0723105 i, \pm 0.16229 \pm$ & 36 \\
& $0.0295239 i, \pm 0.178323 \pm 0.0217879 i, \pm 0.272811, \pm 1.0, \pm 1.0, \pm 1.0)$ & \\
\hline \multirow{2}{*}{5} & $( \pm 0.47428 i, \pm 0.188956 i, \pm 0.22628 \pm 0.228735 i, \pm 0.105228 \pm 0.0783695 i, \pm 0.10314 \pm$ & \\
& $0.0206427 i, \pm 0.129732, \pm 0.141316 \pm 0.0409263 i, \pm 0.138474, \pm 0.152133 \pm$ & \\
& $0.0327313 i, \pm 0.261805, \pm 1.0, \pm 1.0, \pm 1.0)$ & \\
\hline
\end{tabular}

$f(z)=\left(z^{2}-1\right)^{m}$ are found and displayed in Table 4 for values of $2 \leq m \leq 5$.

In the latter part of Section 6, complex dynamics behind the extraneous fixed points will be discussed along with chaotic behavior of rational iterative maps (36) when applied to various polynomials $p(z)$, based on visual description of their basins of attraction along with comparison of their dynamic properties and characteristics.

\section{Numerical Experiment}

We have conducted numerical experiments with a number of test functions using Mathematica Version 7 to confirm the optimal fourth-order convergence. We have assigned 200 significant digits to the minimum number of precision digits and prescribed error bound of $\varepsilon=10^{-150}$ throughout the current experiment. The initial values $x_{0}$ are selected close to the sought zero $\alpha$ for guaranteed convergence to the desired root. All computations have been performed by Mathematica Version 7 with AMD Kaveri 7850 CPU having $3700 \mathrm{Mhz}$ of clock core speed under Windows 7 operating system.

Definition 17 (computational convergence order). Assume that theoretical asymptotic error constant $\eta=$ $\lim _{n \rightarrow \infty}\left(\left|e_{n}\right| /\left|e_{n-1}\right|^{p}\right)$ and convergence order $p \geq 1$ are known. Define $p_{n}=\log \left|e_{n} / \eta\right| / \log \left|e_{n-1}\right|$ as the computational convergence order. Note that $\lim _{n \rightarrow \infty} p_{n}=p$. 
TABLE 5: Convergence for test functions $F_{1}(x)-F_{6}(x)$ with selected methods $Y 1-Y 6$.

\begin{tabular}{|c|c|c|c|c|c|c|c|c|}
\hline MT & $F$ & $n$ & $x_{n}$ & $\left|F\left(x_{n}\right)\right|$ & $\left|x_{n}-\alpha\right|$ & $\left|e_{n} / e_{n-1}^{4}\right|$ & $\eta$ & $p_{n}$ \\
\hline \multirow{5}{*}{$Y 1$} & \multirow{5}{*}{$F_{1}$} & 0 & $0.490000000000000-2.57000000000000 i$ & 0.000678292 & 0.0298039 & & & \\
\hline & & 1 & $0.500000115882539-2.59807622867990 i$ & $1.67 \times 10^{25}$ & $1.17 \times 10^{-7}$ & 0.1484996662 & 0.1356337586 & 3.97420 \\
\hline & & 2 & $0.500000000000000-2.59807621135332 i$ & $3.78 \times 10^{-112}$ & $2.55 \times 10^{-29}$ & 0.1356337326 & & 4.00000 \\
\hline & & 3 & $0.500000000000000-2.59807621135332 i$ & $9.98 \times 10^{-459}$ & $5.79 \times 10^{-116}$ & 0.1356337586 & & 4.00000 \\
\hline & & 4 & $0.500000000000000-2.59807621135332 i$ & $0.0 \times 10^{-795}$ & $0.0 \times 10^{-119}$ & & & \\
\hline \multirow{5}{*}{$Y 2$} & \multirow{5}{*}{$F_{2}$} & 0 & -0.890000000000000 & $3.66 \times 10^{-7}$ & 0.0154886 & & & \\
\hline & & 1 & -0.905488640138161 & $1.61 \times 10^{-47}$ & $2.90 \times 10^{-9}$ & 0.05047432426 & 0.04879773270 & 3.99189 \\
\hline & & 2 & -0.905488637233314 & $4.73 \times 10^{-200}$ & $3.47 \times 10^{-36}$ & 0.04879773239 & & 4.00000 \\
\hline & & 3 & -0.905488637233314 & $3.48 \times 10^{-855}$ & $7.11 \times 10^{-144}$ & 0.04879773270 & & 4.00000 \\
\hline & & 4 & -0.905488637233314 & $0.0 \times 10^{-1196}$ & $0.0 \times 10^{-200}$ & & & \\
\hline \multirow{5}{*}{$Y 3$} & \multirow{5}{*}{$F_{3}$} & 0 & 1.19000000000000 & 0.102779 & 0.190000 & & & \\
\hline & & 1 & 0.999178734348023 & $2.17 \times 10^{13}$ & $0.0008 \times 10^{-13}$ & 0.6301867327 & 0.2584060085 & 3.46320 \\
\hline & & 2 & 0.999999999999883 & $1.27 \times 10^{-62}$ & $1.17 \times 10^{-53}$ & 0.2572719538 & & 4.00062 \\
\hline & & 3 & 1.00000000000000 & $1.55 \times 10^{-259}$ & $4.84 \times 10^{-53}$ & 0.2584060085 & & 4.00000 \\
\hline & & 4 & 1.00000000000000 & $0.0 \times 10^{-1000}$ & $0.0 \times 10^{-200}$ & & & \\
\hline \multirow{5}{*}{$Y 4$} & \multirow{5}{*}{$F_{4}$} & 0 & 2.65000000000000 & 0.486088 & 0.00424869 & & & \\
\hline & & 1 & 2.64575131054487 & $8.73 \times 10^{-22}$ & $5.19 \times 10^{-10}$ & 1.594967181 & 1.992474266 & 4.04075 \\
\hline & & 2 & 2.64575131106459 & $1.91 \times 10^{-104}$ & $1.45 \times 10^{-37}$ & 1.992474316 & & 4.00000 \\
\hline & & 3 & 2.64575131106459 & $4.38 \times 10^{-435}$ & $8.89 \times 10^{-148}$ & 1.992474266 & & 4.00000 \\
\hline & & 4 & 2.64575131106459 & $0.0 \times 10^{-593}$ & $0.0 \times 10^{-199}$ & & & \\
\hline \multirow{5}{*}{$Y 5$} & \multirow{5}{*}{$F_{5}$} & 0 & 1.19000000000000 & $1.41 \times 10^{12}$ & 0.0215466 & & & \\
\hline & & 1 & 1.06845276583309 & $2.37 \times 10^{-53}$ & $6.27 \times 10^{-7}$ & 2.911081724 & 2.437575882 & 3.95374 \\
\hline & & 2 & 1.06845339326982 & $2.47 \times 10^{-217}$ & $3.77 \times 10^{-25}$ & 2.4375758821 & & 4.00000 \\
\hline & & 3 & 1.06845339326982 & $2.89 \times 10^{-873}$ & $4.96 \times 10^{-98}$ & 2.437575882 & & 4.00000 \\
\hline & & 4 & 1.06845339326982 & $0.0 \times 10^{-1799}$ & $0.0 \times 10^{-199}$ & & & \\
\hline \multirow{5}{*}{$Y 6$} & \multirow{5}{*}{$F_{6}$} & 0 & 1.89000000000000 & 0.0000253422 & 0.0229312 & & & \\
\hline & & 1 & 1.91292827592121 & $1.07 \times 10^{-36}$ & $2.90 \times 10^{-6}$ & 10.51276543 & 14.87454509 & 4.09193 \\
\hline & & 2 & 1.91293118277239 & $3.41 \times 10^{-160}$ & $1.06 \times 10^{-21}$ & 14.87411967 & & 4.00000 \\
\hline & & 3 & 1.91293118277239 & $3.46 \times 10^{-654}$ & $1.89 \times 10^{-83}$ & 14.87454509 & & 4.00000 \\
\hline & & 4 & 1.91293118277239 & $0.0 \times 10^{-1597}$ & $0.0 \times 10^{-199}$ & & & \\
\hline
\end{tabular}

Typical methods $Y 1, Y 2, \ldots, Y 6$ have been applied to the test functions $F_{1}-F_{6}$ below:

$$
\begin{aligned}
& F_{1}(x)=\left(1+e^{-2 x}\right)\left(x^{2}-x+7\right)^{4}, \\
& \qquad m=4, \alpha=\frac{1-3 \sqrt{3} i}{2}, i=\sqrt{-1}, \\
& F_{2}(x)=\left(4+3 \sin x-2 x^{2}\right)^{6}, \quad m=6, \alpha \approx-0.9, \\
& F_{3}(x)=\left(\cos \left(\frac{\pi}{2} x\right)+e^{1-x^{2}}-1\right)^{5}, \quad m=5, \alpha=1, \\
& F_{4}(x)=e^{x^{2}+x+1}\left(x^{2}-7\right)^{2} \log \left(x^{2}-6\right), \\
& F_{5}(x)=\left(\cos \left(x^{2}+x\right)-x \log \left(x^{2}-\pi+3\right)+1\right)^{8} \\
& \quad \cdot\left(x^{2}+2-\pi\right), \quad m=9, \alpha=\sqrt{\pi-2},
\end{aligned}
$$

$$
\begin{array}{r}
F_{6}(x)=\log ^{4}\left(x^{3}-6\right) \cos \left(x^{3}-7+\frac{\pi}{2}\right) \log \left(x^{3}-7\right), \\
m=8, \alpha=7^{1 / 3} .
\end{array}
$$

As seen in Table 5, the order of convergence is four and the values of computational asymptotic error constant well approach theoretical value $\eta$.

The following test functions $f_{1}, f_{2}, \ldots, f_{7}$ listed below further confirm the convergence behavior of our proposed methods (5). Consider

$$
\begin{aligned}
f_{1}(x) & =x^{9}+4 x^{7}-5, \\
\alpha & =-0.92+0.39 i, m=1, x_{0}=-0.91+0.41 i, \\
f_{2}(x) & =\left(e^{x^{6}-x^{3}-7}-1\right)\left(x^{6}-x^{3}-7\right), \\
\alpha & =\left(\frac{1+\sqrt{29}}{2}\right)^{1 / 3}=1.47, m=2, x_{0}=1.46,
\end{aligned}
$$


TABLE 6: Comparison of $\left|x_{n}-\alpha\right|$ for high-order iterative methods.

\begin{tabular}{|c|c|c|c|c|c|c|c|c|c|c|c|}
\hline$f(x)$ & $\lambda$ & $c_{n}-\alpha \mid$ & Kan & Sol & $\mathrm{Li}$ & $Y 1$ & $Y 2$ & $Y 3$ & $Y 4$ & Y5 & $Y 6$ \\
\hline \multirow{3}{*}{$f_{1}$} & \multirow{3}{*}{$-0.91+0.41 i$} & $\left|x_{1}-\alpha\right|$ & $3.02 e-5^{*}$ & $.05 e-5$ & $7.1 e-6$ & $1.67 e-5$ & $1.67 e-5$ & $2.17 e-5$ & $2.52 e-7$ & $2.92 e-5$ & $2.17 e-5$ \\
\hline & & $\left|x_{2}-\alpha\right|$ & $5.49 e-17$ & $2.13 e-16$ & $3.69 e-20$ & $2.66 e-18$ & $2.66 e-18$ & $9.63 e-18$ & $1.19 e-27$ & $4.2 e-17$ & $9.63 e-18$ \\
\hline & & $\left|x_{3}-\alpha\right|$ & $6.00 e-64$ & $64 e-61$ & $2.71 e-77$ & $.71 e-69$ & $1.71 e-69$ & $3.73 e-67$ & $6.11 \mathrm{e}-109$ & $1.8 e-64$ & $3.73 e-67$ \\
\hline \multirow{3}{*}{$f_{2}$} & \multirow{3}{*}{1.46} & $\left|x_{1}-\alpha\right|$ & 305 & $.99 e-5$ & 00 & $2.88 e-5$ & $2.88 e-5$ & $17 \rho-5$ & $8.65 e-5$ & $7.36 e-5$ & $3.43 e-5$ \\
\hline & & $\left|x_{2}-\alpha\right|$ & $1.55 e-15$ & $1.21 e-16$ & $1.21 e-16$ & $7.92 e-16$ & $7.92 e-16$ & $1.27 e-15$ & $3.67 e-11$ & $9.57 e-15$ & $1.90 e-15$ \\
\hline & & $\left|x_{3}-\alpha\right|$ & $7.65 e-57$ & $1.67 e-61$ & $1.67 e-61$ & $4.52 e-58$ & $4.52 e-58$ & $3.38 e-57$ & & $2.73 e-54$ & $1.79 e-56$ \\
\hline \multirow{3}{*}{$f_{3}$} & \multirow{3}{*}{-2.61} & & & & & & & $1.09 e-5$ & & & $1.09 e-5$ \\
\hline & & $\left|x_{2}-\alpha\right|$ & $6.94 e-20$ & $8.08 e-20$ & $7.46 e-20$ & $6.94 e-20$ & $6.94 e-20$ & $6.83 e-20$ & $6.08 e-20$ & $6.76 e-20$ & $6.83 e-20$ \\
\hline & & $\left|x_{3}-\alpha\right|$ & $1.10 e-76$ & $2.15 e-76$ & $1.51 e-76$ & $1.10 e-76$ & $1.10 e-76$ & $1.03 e-76$ & $6.17 e-77$ & $9.84 e-77$ & $1.03 e-76$ \\
\hline \multirow{3}{*}{$f_{4}$} & \multirow{3}{*}{$0.49-2.25 i$} & $\left|x_{1}-\alpha\right|$ & $1.09 e-5$ & $3.78 e-6$ & $4.11 e-6$ & $4.29 e-6$ & $4.29 e-6$ & $432 e-6$ & $4.44 e-6$ & $4.33 e-6$ & $4.32 e-6$ \\
\hline & & $\left|x_{2}-\alpha\right|$ & $6.94 e-20$ & $6.67 e-24$ & $1.01 e-23$ & $1.26 e-23$ & $1.26 e-23$ & $1.30 e-23$ & $1.50 e-23$ & $1.32 e-23$ & $1.30 e-23$ \\
\hline & & $\left|x_{3}-\alpha\right|$ & $1.10 e-76$ & $6.46 \mathrm{e}-95$ & $3.82 e-94$ & $9.42 e-94$ & $9.42 e-94$ & $1.07 e-93$ & $1.99 e-93$ & $1.17 e-93$ & $1.07 e-93$ \\
\hline \multirow{3}{*}{$f_{5}$} & \multirow{3}{*}{9.03} & $\left|x_{1}-\alpha\right|$ & $1.09 e-5$ & $4.50 e-5$ & $4.47 e-5$ & $4.45 e-5$ & $4.45 e-5$ & $4.45 e-5$ & $4.45 e-5$ & $4.45 e-5$ & $4.45 e-5$ \\
\hline & & $\left|x_{2}-\alpha\right|$ & $6.94 e-20$ & $3.27 e-16$ & $3.17 e-16$ & $3.13 e-16$ & $3.13 e-16$ & $3.13 e-16$ & $3.11 e-16$ & $3.13 e-16$ & $3.13 e-16$ \\
\hline & & $\left|x_{3}-\alpha\right|$ & $1.10 \mathrm{e}-76$ & $9.08 e-61$ & $8.06 e-61$ & $7.70 e-61$ & $7.70 e-61$ & $7.65 e-61$ & $7.50 e-61$ & $7.63 e-61$ & $7.65 e-61$ \\
\hline \multirow{3}{*}{$f_{6}$} & \multirow{3}{*}{$-2.19 i$} & $\left|x_{1}-\alpha\right|$ & $1.09 e-5$ & $1.72 e-4$ & $1.53 e-4$ & $1.56 e-4$ & $1.56 e-4$ & $1.56 e-4$ & $1.58 e-4$ & $1.57 e-4$ & $1.56 e-4$ \\
\hline & & $\left|x_{2}-\alpha\right|$ & $6.94 e-20$ & $6.35 e-15$ & $5.99 e-15$ & $7.02 e-15$ & $7.02 e-15$ & $7.18 e-15$ & $7.73 e-15$ & $7.27 e-15$ & $7.18 e-15$ \\
\hline & & $\left|x_{3}-\alpha\right|$ & $1.10 \mathrm{e}-76$ & $1.18 e-56$ & $1.37 e-56$ & $2.88 e-56$ & $2.88 e-56$ & $3.17 e-56$ & $4.39 e-56$ & $3.35 e-56$ & $3.17 e-56$ \\
\hline
\end{tabular}

${ }^{*} 3.02 e-5=3.02 \times 10^{-5}$.

$$
\begin{gathered}
f_{3}(x)=e^{x^{2}+1}\left(x^{2}-7\right)^{2} \sec x \log \left(x^{2}-6\right), \\
\alpha=-\sqrt{7}=-2.64575, m=3, x_{0}=-2.59, \\
f_{4}(x)=\left(5-x+x^{2}\right)^{4} \cot \left(x^{3}+7\right), \\
\alpha=\frac{1-\sqrt{19} i}{2}, m=4, x_{0}=0.49-2.25 i, \\
f_{5}(x)=\left(x^{5}-9 x^{4}+3 x-27\right) e^{x^{2}+x} \sin \left(\frac{\pi x}{9}\right) \\
\cdot\left(\log \left(x^{2}-6 x-26\right)\right)^{3}, \\
\alpha=9, m=5, x_{0}=9.12, \\
f_{6}(x)=\left(x^{2}+5\right)^{4}\left(\log \left(x^{3}-3 x^{2}+5 x-14\right)\right)^{2}, \\
\alpha=-\sqrt{5} i, m=6, x_{0}=-2.18 i .
\end{gathered}
$$

Table 6 shows the comparison of $\left|x_{n}-\alpha\right|$ among listed methods Kan, Sol, and Li and Y1-Y6 described in Section 1. In Table 6, the least errors within the prescribed error bound are highlighted in bold face. Although we are limited to the selected current experiments, within three iterations, a strict comparison shows that method Y4 displays slightly better convergence for test functions $f_{1}$ and $f_{3}$, and method Sol displays slightly better convergence for test function $f_{4}$, while method Kan displays slightly better convergence for test functions $f_{5}$ and $f_{6}$. In addition, both methods Sol and $\mathrm{Li}$ show similar convergence for test function $f_{2}$. If we closely view the definition of the asymptotic error constant, we will find that the local convergence is dependent on the function $f(x)$, an initial guess $x_{0}$, the multiplicity $m$, and zero $\alpha$. Consequently, for a given set of test functions, one method is hardly expected to always show better performance than the others.

It is important to properly select initial values guaranteeing the convergence of iterative schemes. For ensured convergence of iterative map (5), it requires good initial values close to zero $\alpha$. It is not easy to determine how close the initial values are to zero $\alpha$, since initial values are generally dependent upon computational precision, error bound, and the given function $f(x)$ under consideration. One efficient way of selecting stable initial guesses is to directly use visual basins of attraction [27, 35, 36]. Since the area of convergence can be seen on the basins of attraction, it would be a reasonable measure of convergence behavior. One would say that a method having a larger area of convergence implies a more stable method. Obviously a quantitative analysis becomes an essential tool for measuring the size of area of convergence. In the next section, we will illustrate the basins of attraction of the listed methods when applied to a variety of polynomials with multiple zeros and discuss underlying relevant dynamics.

\section{Basins of Attraction}

Throughout the current dynamics experiment, for effective constructing basins of attraction, we have employed a tolerance $\varepsilon=10^{-4}$ for convergence within a maximum of 40 iterations. To illustrate the desired basins of attraction, we first take a $600 \times 600$ uniform grid point in a $6 \times 6$ square region 


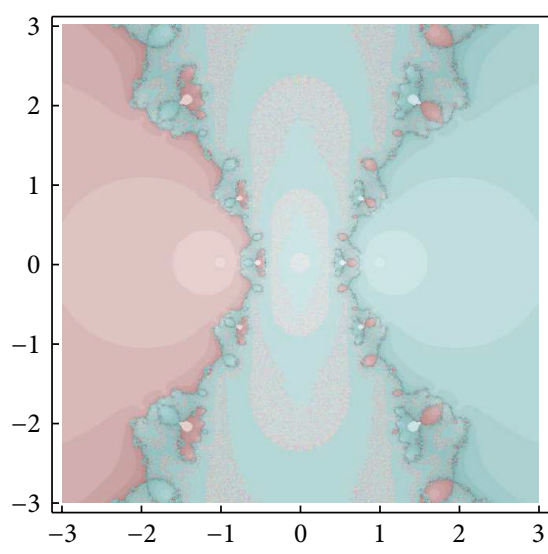

(a)

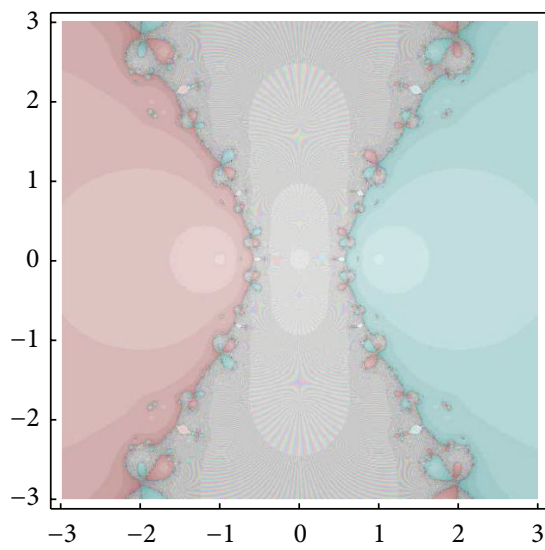

(d)

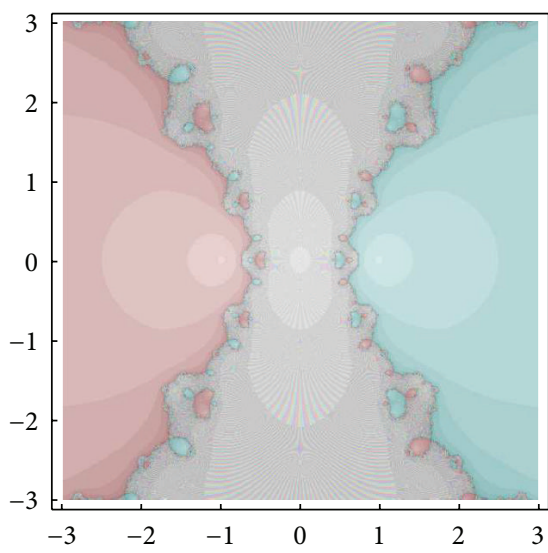

(g)

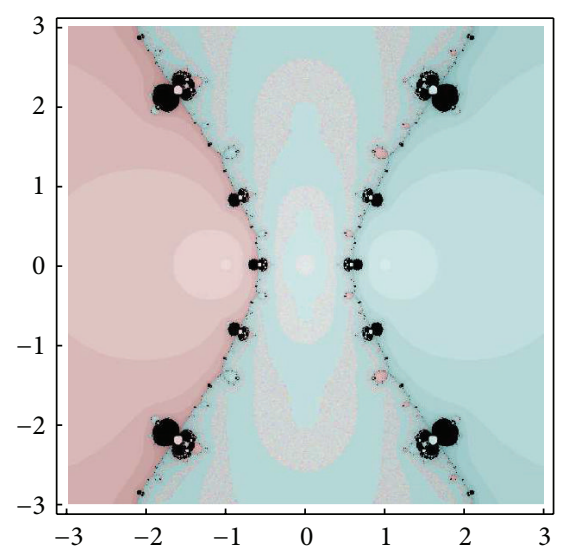

(b)

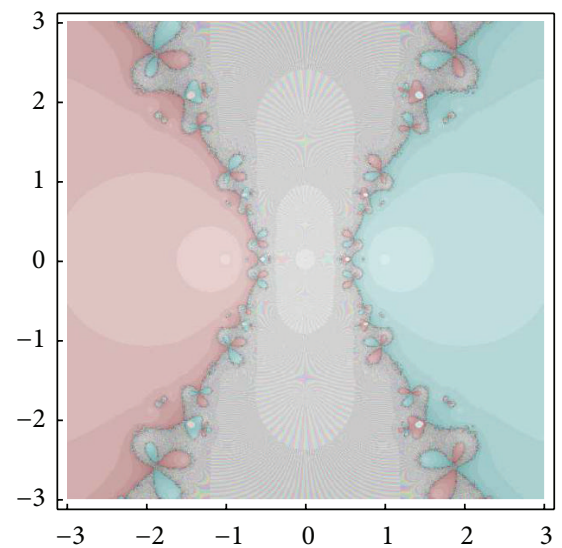

(e)

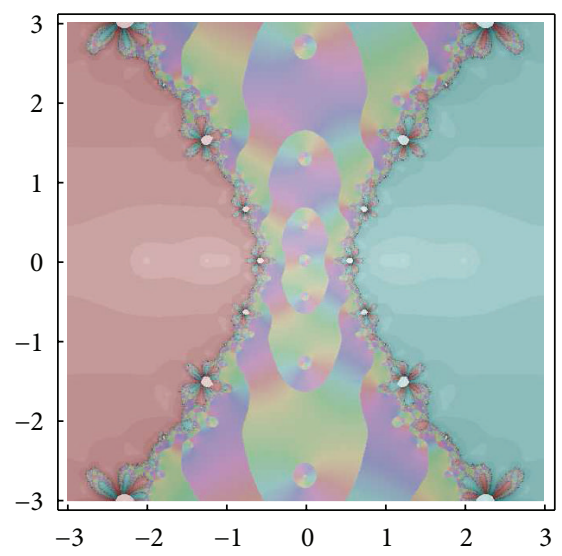

(h)

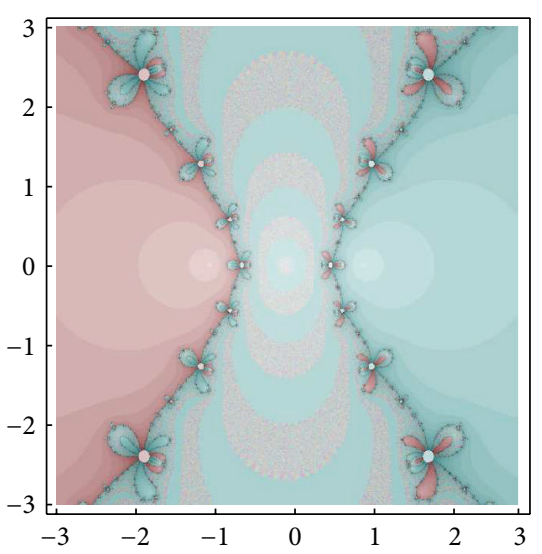

(c)

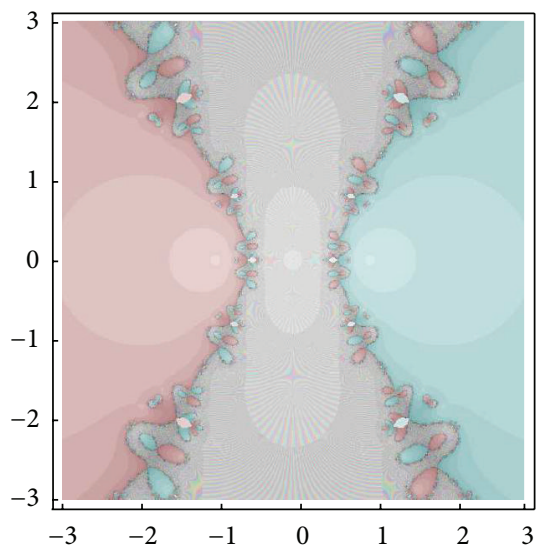

(f)

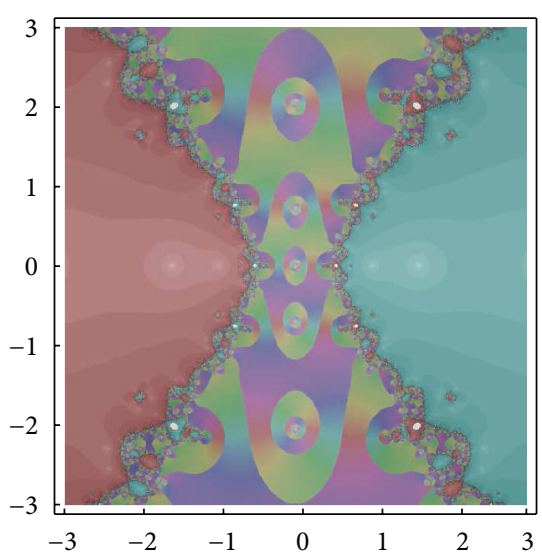

(i)

Figure 3: (a) Kan, (b) Sol, (c) Zhou, (d) Y1, (e) Y2, (f) Y3, (g) Y4, (h) Y5, and (i) Y6, for the roots of the polynomial $P_{1}(z)=\left(z^{3}-z\right)^{2}$.

centered at the origin of the complex plane, which contains all roots of test functions selected. We then paint the initial points on the basins of attraction, with a diversity of colors ranging from bright ones to dark ones based on the iteration number for convergence. In Figures 3-8, the black points are regarded as the points for which the corresponding iteration scheme starting from an initial point does not converge to any root of the test polynomial under consideration. We have applied all the methods mentioned in Section 1 to a variety of polynomials having multiple roots with multiplicity of $m=$ 2, 3, 4, 5, 6. In Tables 7-12, abbreviations CPU, TCON, AVG, and TDIV denote CPU time measured in units of seconds for convergence, the number of total convergent points, the number of average iteration for convergence, and the number 


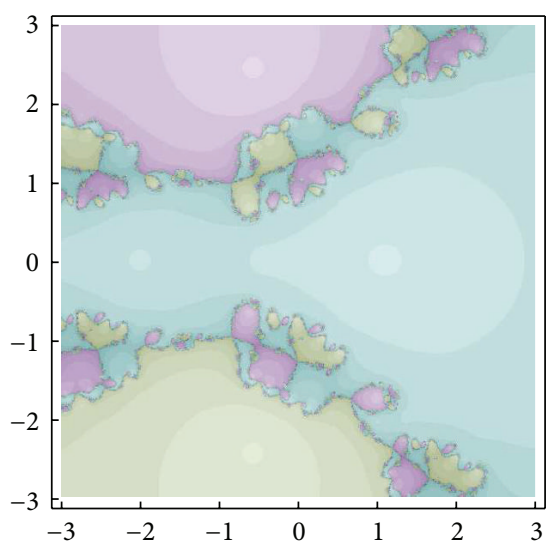

(a)

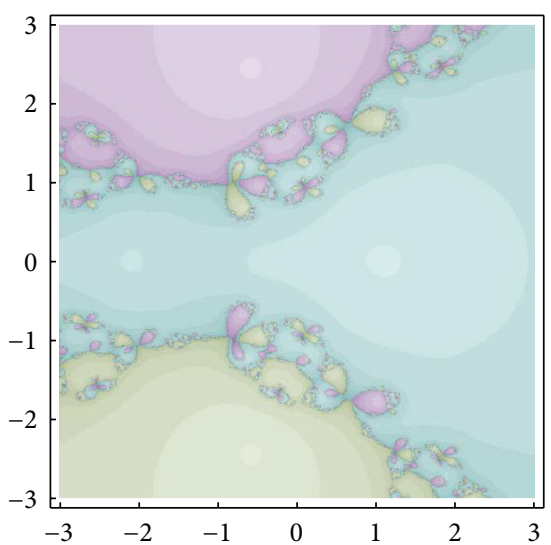

(d)

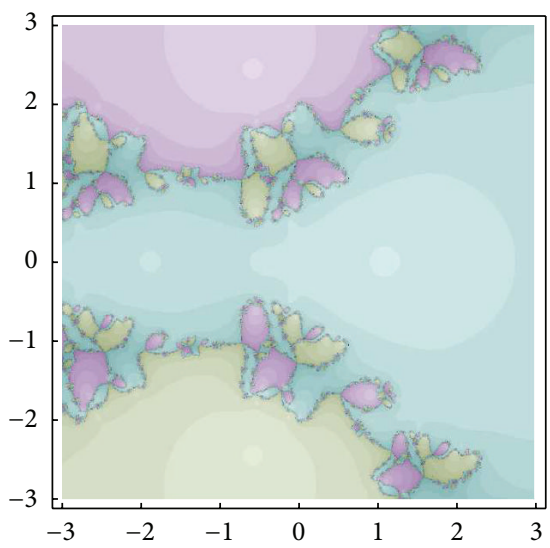

(g)

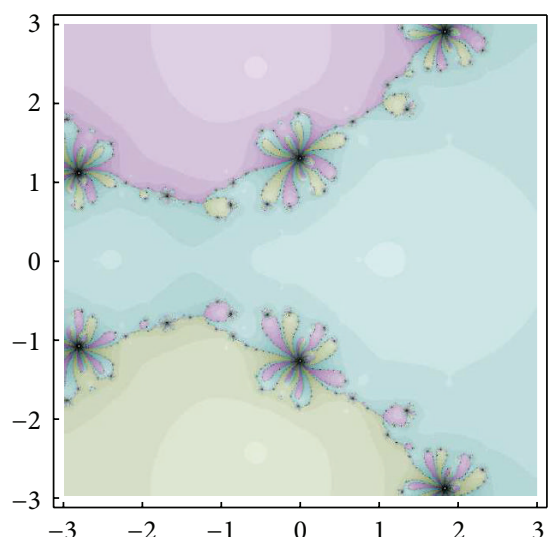

(b)

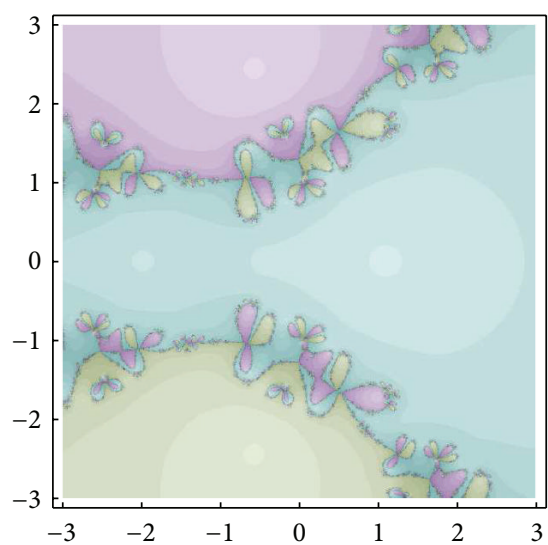

(e)

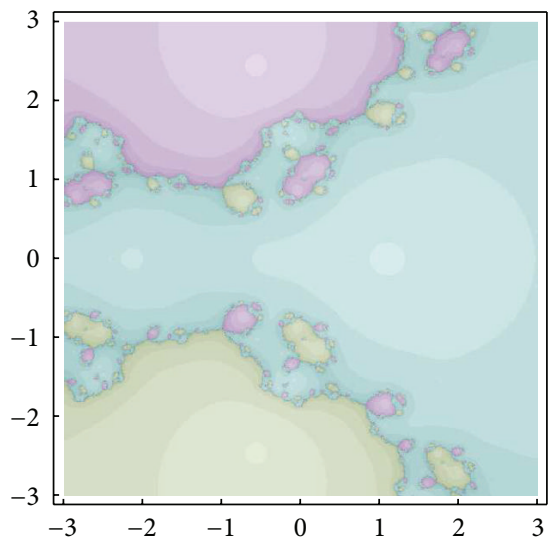

(h)

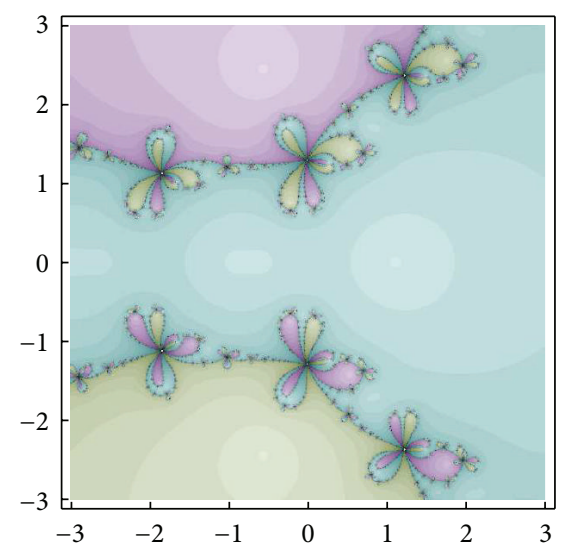

(c)

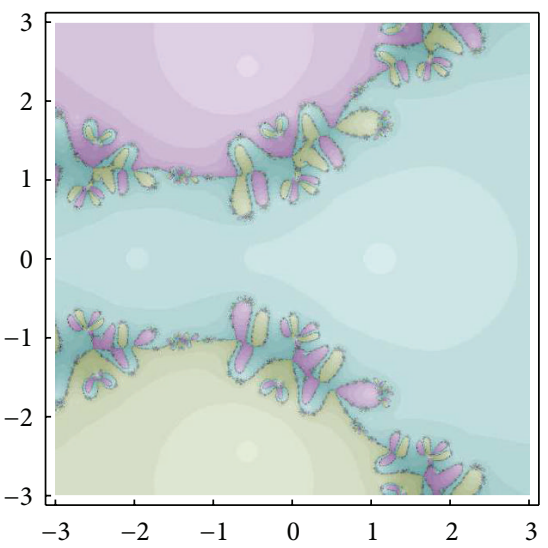

(f)

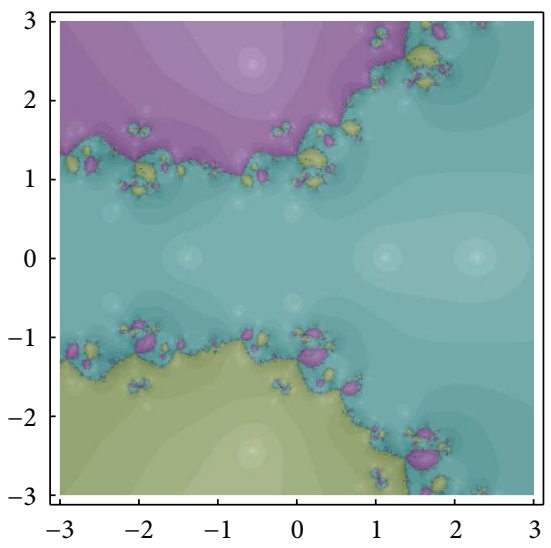

(i)

Figure 4: (a) Kan, (b) Sol, (c) Zhou, (d) Y1, (e) Y2, (f) Y3, (g) Y4, (h) Y5, and (i) Y6, for the roots of the polynomial $P_{2}(z)=\left(z^{3}+5 z-7\right)^{3}$.

of divergent points, respectively. At this point, we now begin by presenting various examples to display the desired basins of attraction.

As a first example, we have taken the following polynomial:

$$
P_{1}(z)=\left(z^{3}-z\right)^{2}
$$

whose roots $z=0, \pm 1$ are all real with multiplicity $m=2$. Based on Table 7 and Figure 3, we find that $Y 1$ has shown best AVG and TDIV, followed by Kan and Y2. As can be seen in Figure 3, Sol has shown considerable amount of black points. These points causing divergence behavior were observed from the last column of Table 7 . The best result for $\mathrm{CPU}$ is given by Zhou and the worst one is given by $Y 6$.

Our next sample has triple roots. The polynomial

$$
P_{2}(z)=\left(z^{3}+5 z-7\right)^{3}
$$




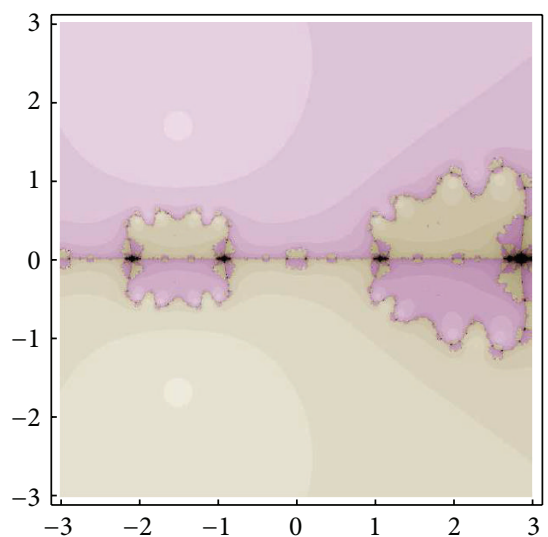

(a)

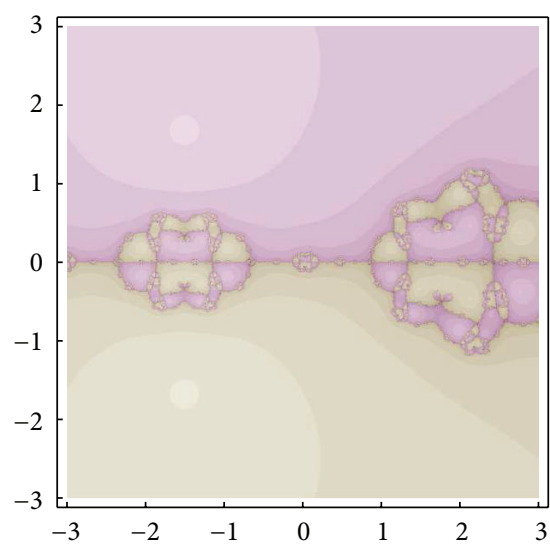

(d)

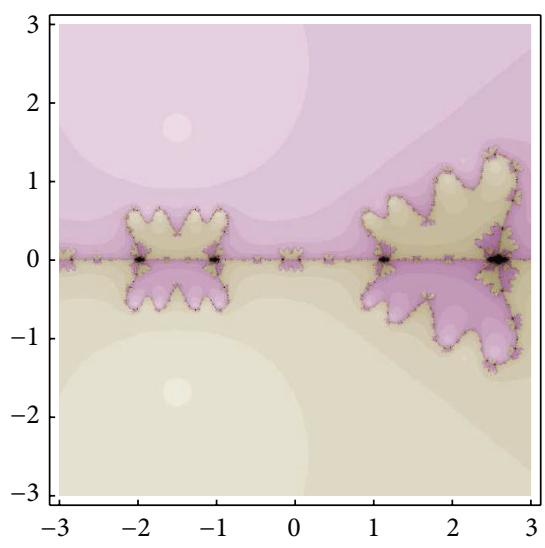

(g)

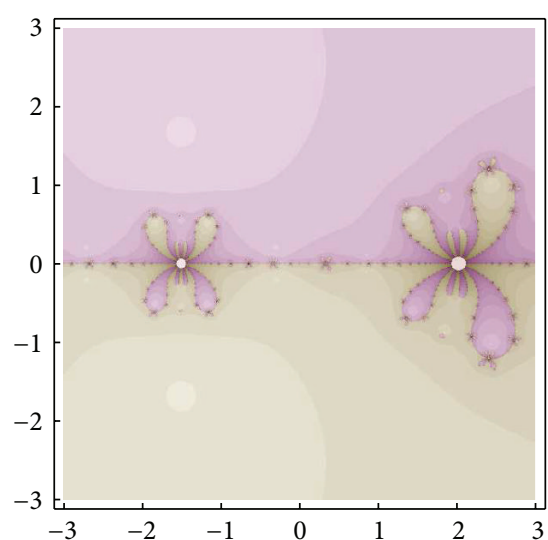

(b)

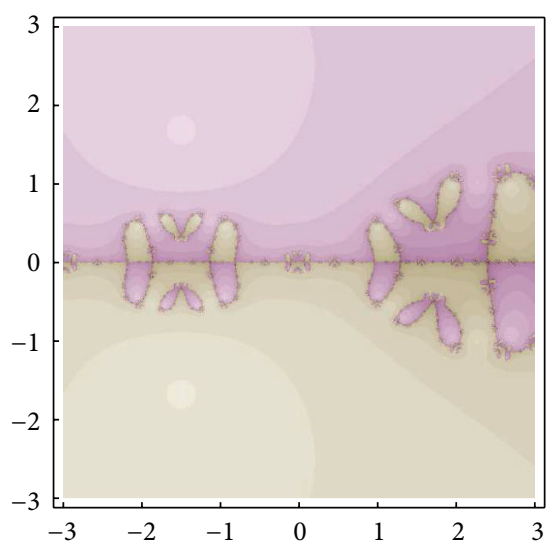

(e)

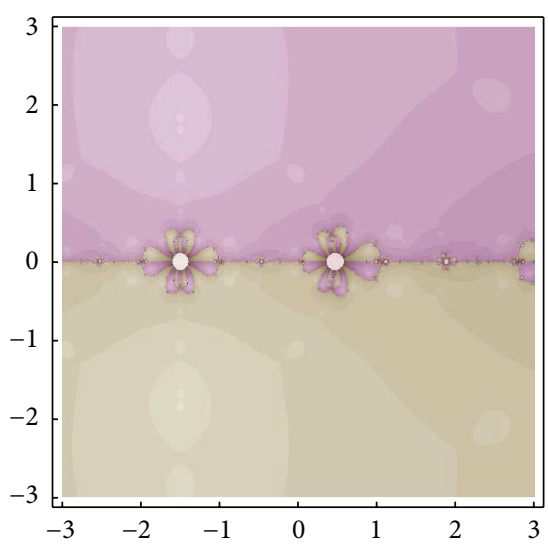

(h)

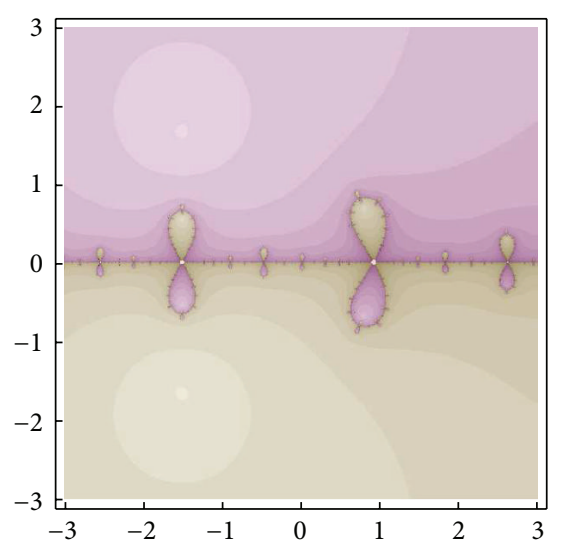

(c)

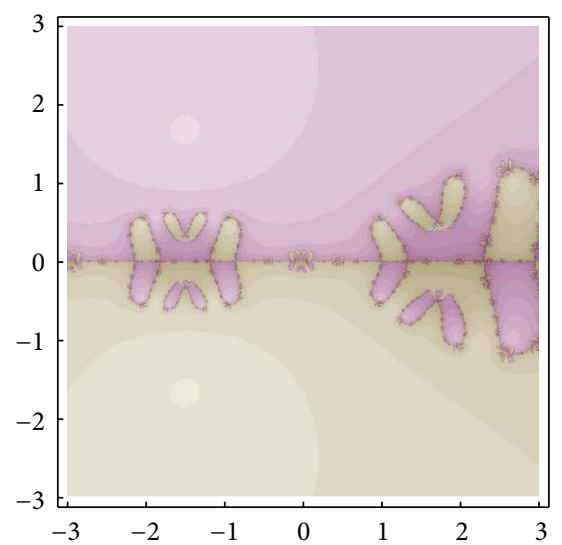

(f)

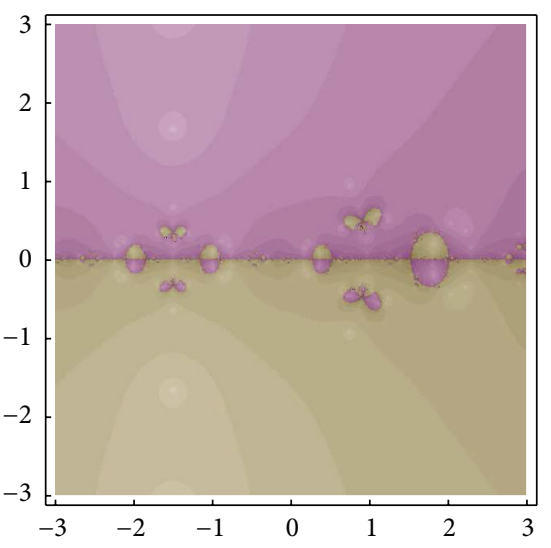

(i)

Figure 5: (a) Kan, (b) Sol, (c) Zhou, (d) Y1, (e) Y2, (f) Y3, (g) Y4, (h) Y5, and (i) Y6, for the roots of the polynomial $P_{3}(z)=\left(z^{2}+3 z+5\right)^{4}$.

has three roots $z=-0.559719-2.43718 i,-0.559719+$ $2.43718 i, 1.11944$ of multiplicity 3 . The statistical results are listed in Table 8 and relevant basins of attraction are illustrated in Figure 4. The method Y5 has performed best AVG and TDIV. As can be seen in Figure 4, Sol has shown considerable amount of black points, while Zhou has shown a few black ones. The best result for CPU is given by $Y 5$ and the worst one is given by $Y 6$.
As a third example, we have taken the following polynomial whose roots are all of multiplicity four:

$$
P_{3}(z)=\left(z^{2}+3 z+5\right)^{4}
$$

whose roots are $z=-1.5-1.65831 i,-1.5+1.65831 i$. The statistical results are presented in Table 9 and relevant basins of attraction are illustrated in Figure 5. The method $Y 1$ has 


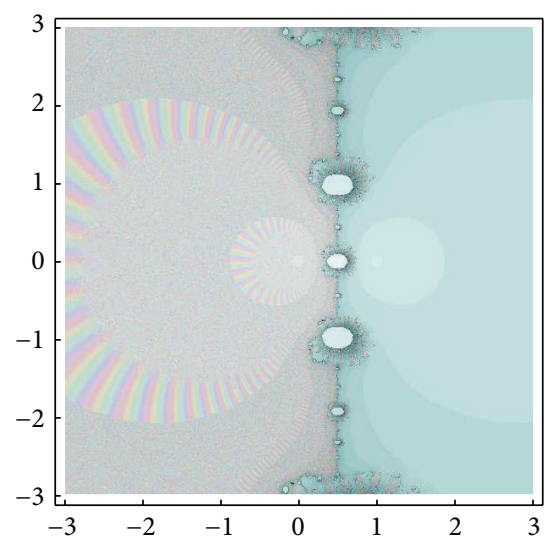

(a)

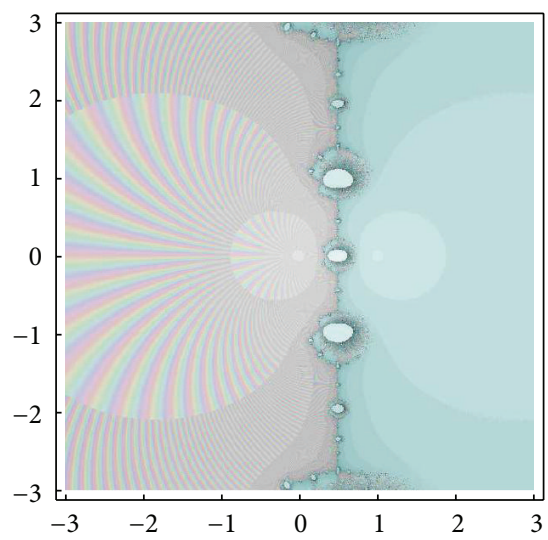

(d)

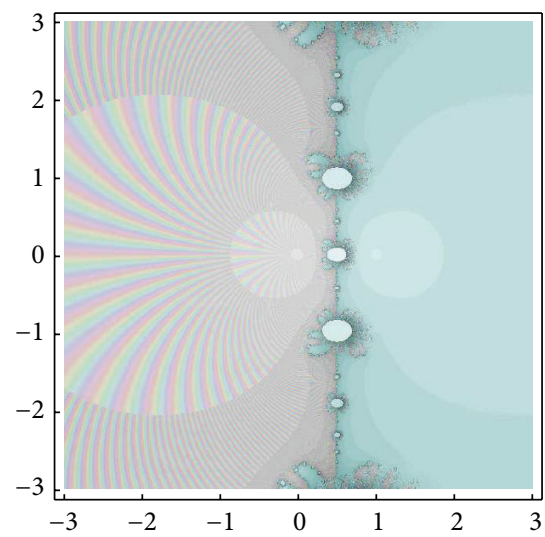

(g)

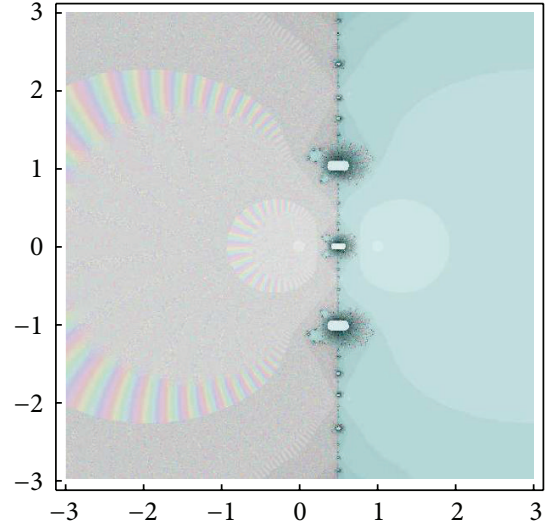

(b)

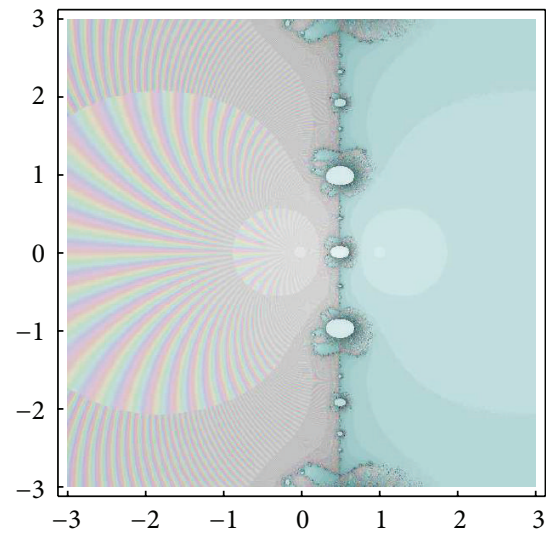

(e)

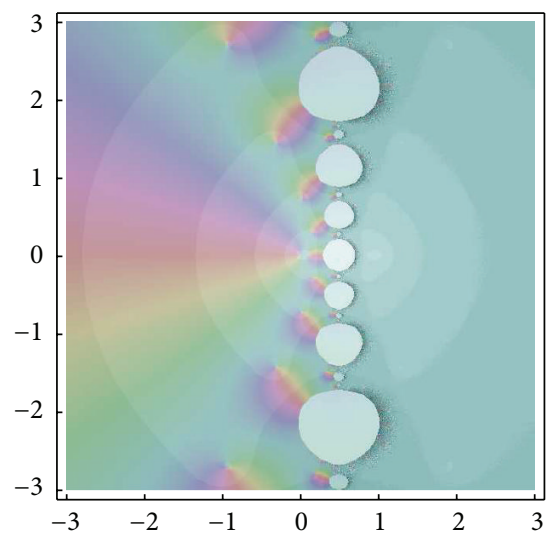

(h)

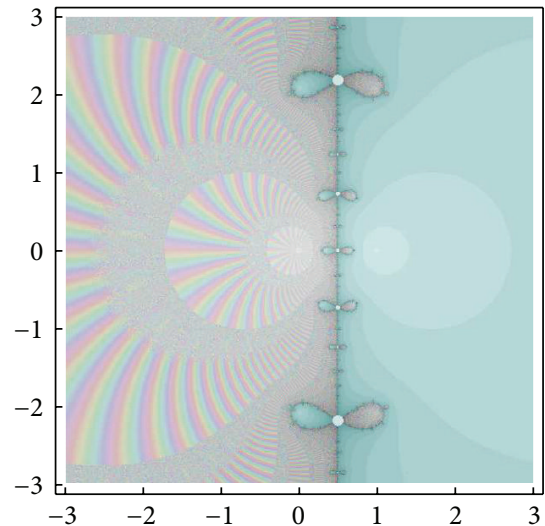

(c)

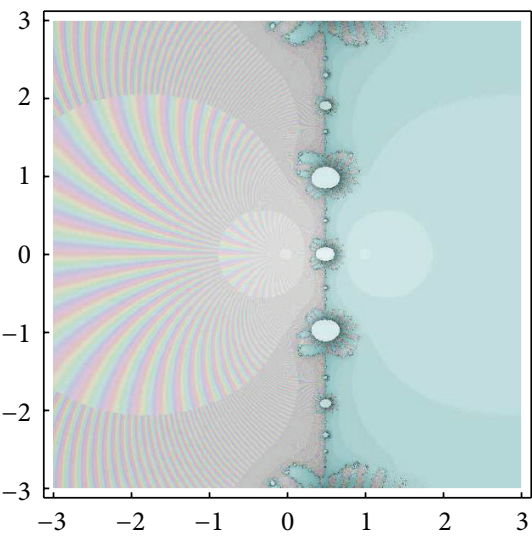

(f)

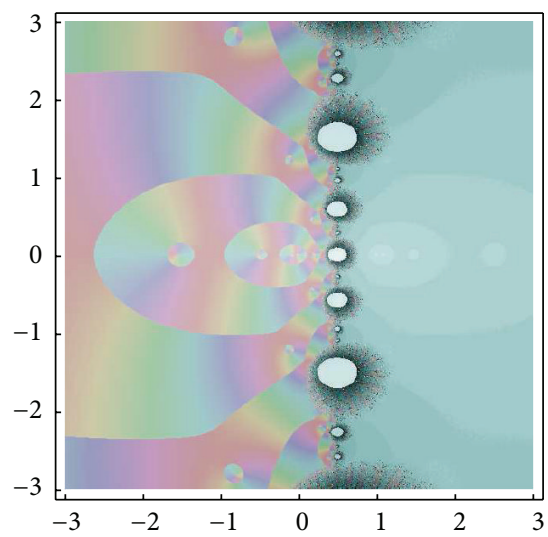

(i)

Figure 6: (a) Kan, (b) Sol, (c) Zhou, (d) Y1, (e) Y2, (f) Y3, (g) Y4, (h) Y5, and (i) Y6, for the roots of the polynomial $P_{4}(z)=\left(z^{2}-z\right)^{6}$.

shown best AVG and TDIV. As can be seen in Figure 5, Kan and $Y 4$ have shown considerable amount of black points, while $Y 5$ and $Y 6$ have shown a few black ones. The best result for CPU is given by Zhou and the worst one is given by $Y 2$.

In the fourth example, we have taken the polynomial having two roots of unity

$$
P_{4}(z)=\left(z^{2}-z\right)^{6} .
$$

The statistical results are presented in Table 10 and relevant basins of attraction are illustrated in Figure 6. The method $Y 1$ has shown best AVG and TDIV. As can be seen in Figure 6, $Y 6$ has shown considerable amount of black points, while Kan and Sol have shown a few black ones. We find that Zhou shows the best result for CPU, while $Y 5$ shows the worst one.

In the fifth example, we have chosen the following polynomial:

$$
P_{5}(z)=\left(z^{2}-1\right)^{2} .
$$




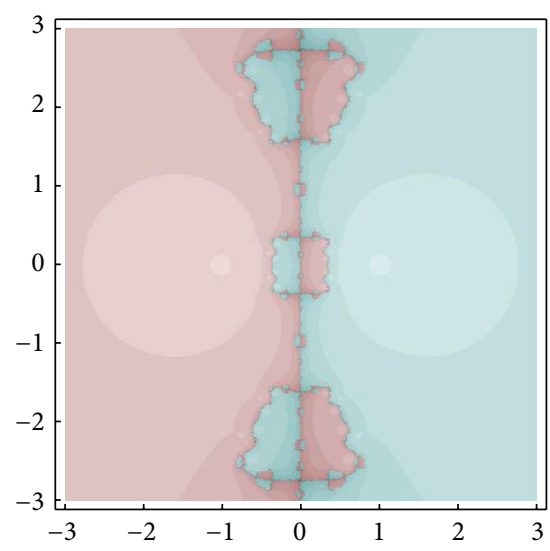

(a)

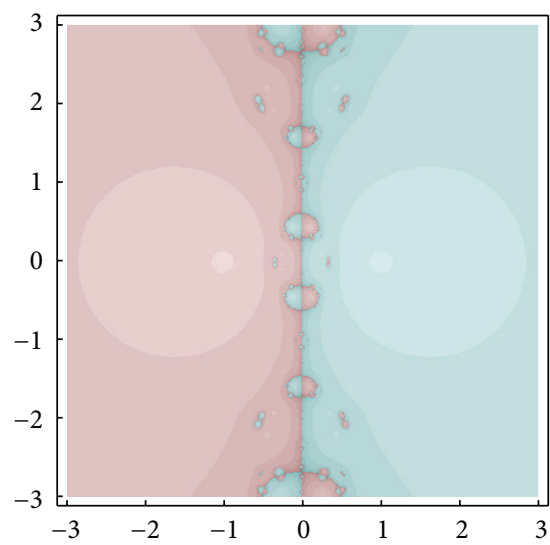

(d)

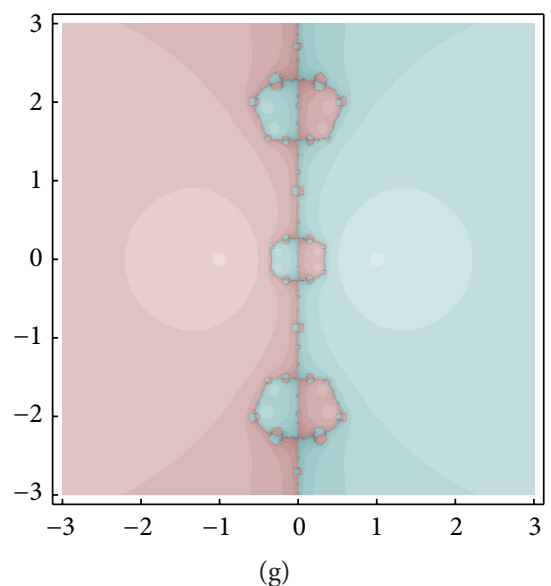

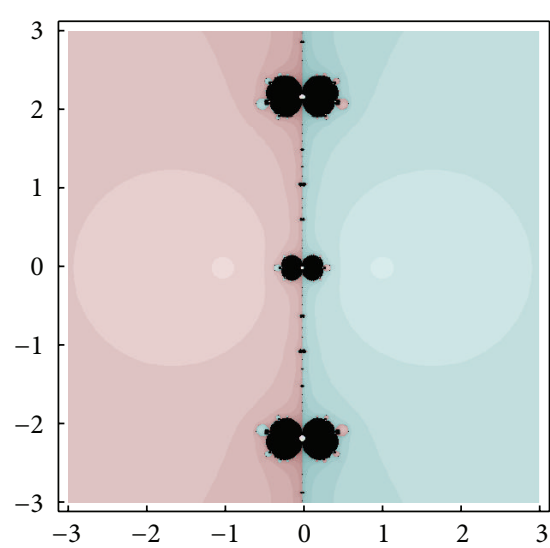

(b)

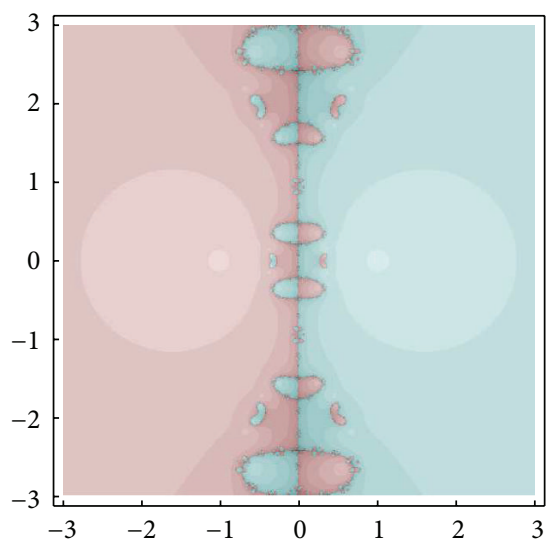

(e)

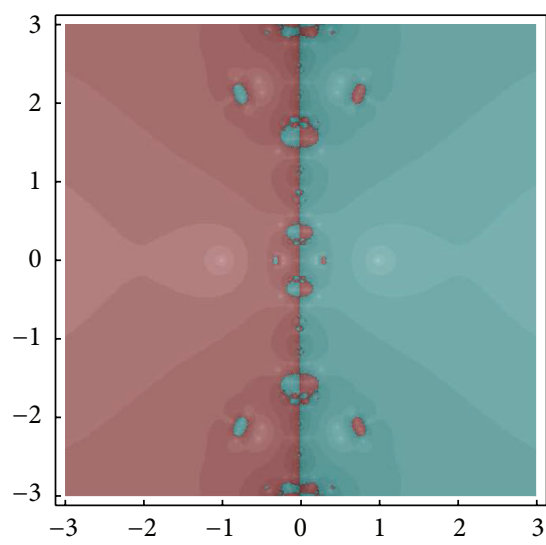

(h)

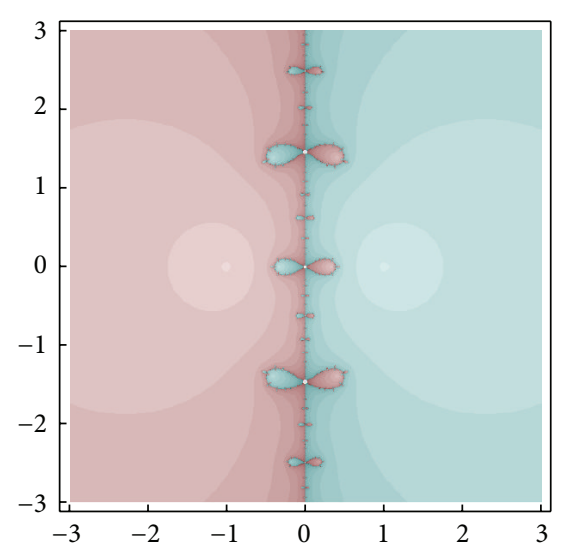

(c)

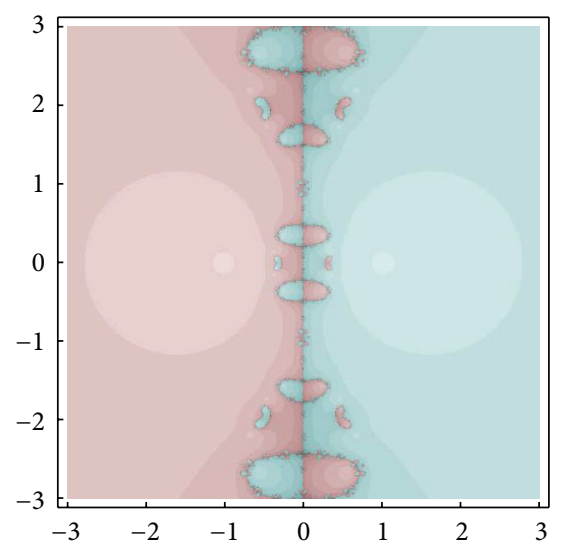

(f)

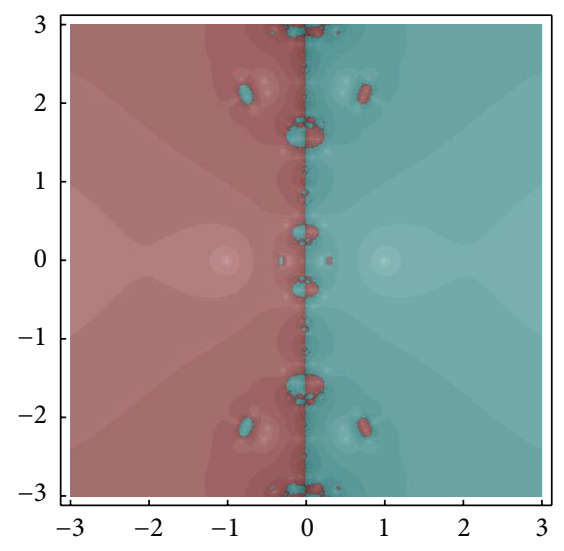

(i)

Figure 7: (a) Kan, (b) Sol, (c) Zhou, (d) Y1, (e) Y2, (f) Y3, (g) Y4, (h) Y5, and (i) Y6, for the roots of the polynomial $P_{4}(z)=\left(z^{2}-1\right)^{2}$.

The statistical results are presented in Table 11 and relevant basins of attraction are displayed in Figure 7. The method $Y 3$ has shown best AVG and TDIV. As can be seen in Figure 7, Sol has shown considerable amount of black points. We find that Zhou shows the best result for CPU, while $Y 5$ shows the worst one.
In the last example, we have selected the polynomial whose roots are all of multiplicity five

$$
P_{6}(z)=\left(z^{2}-1\right)^{5}
$$

The experimented results are presented in Table 12 and relevant basins of attraction are shown in Figure 8. The 


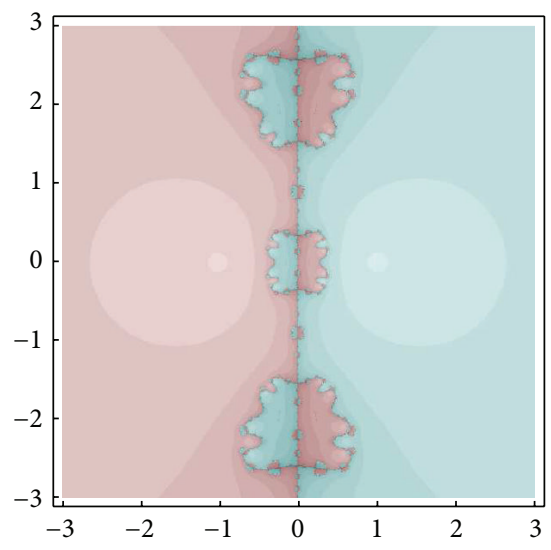

(a)

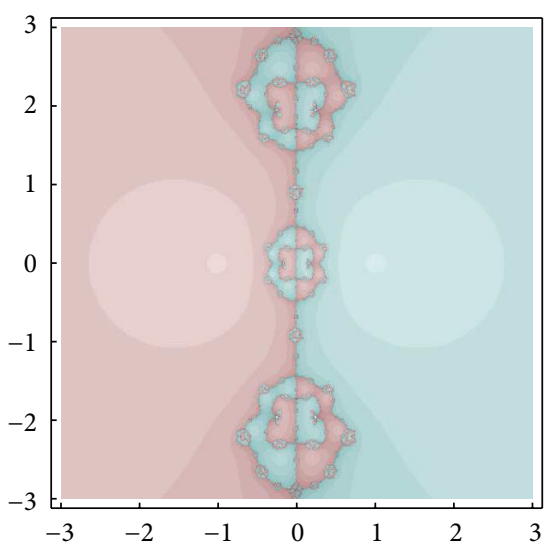

(d)

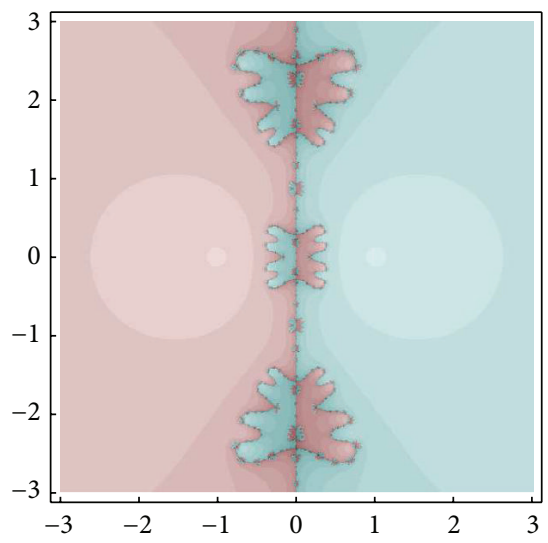

(g)

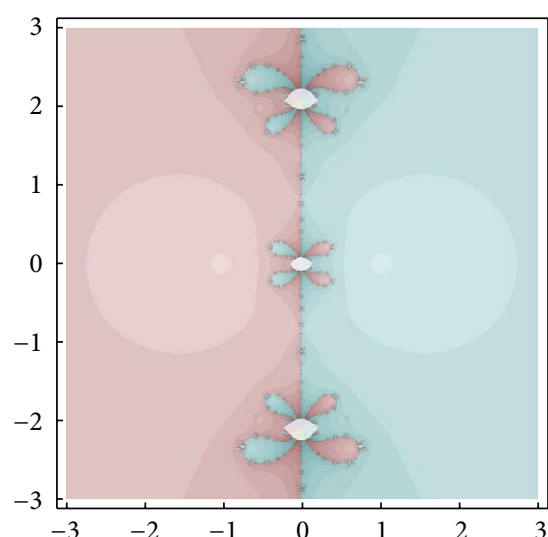

(b)

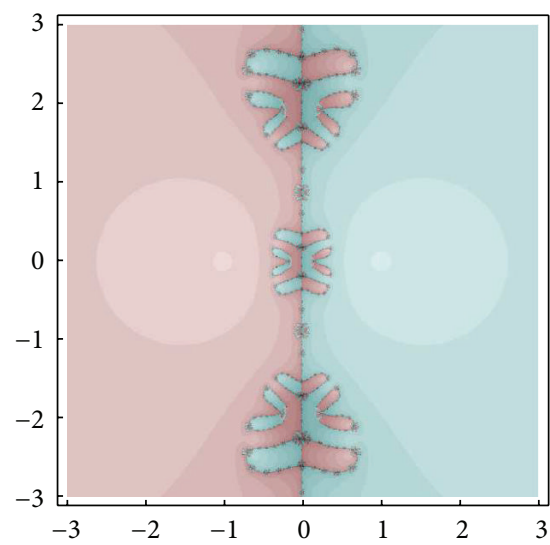

(e)

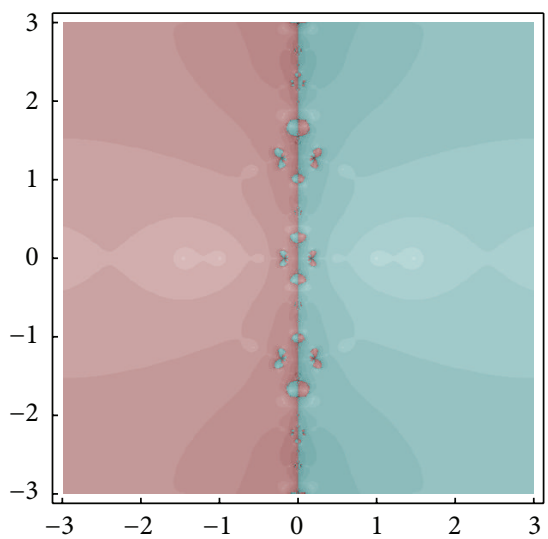

(h)

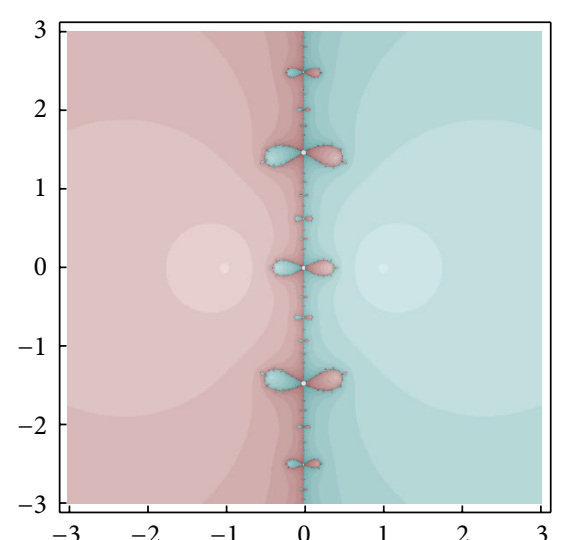

(c)

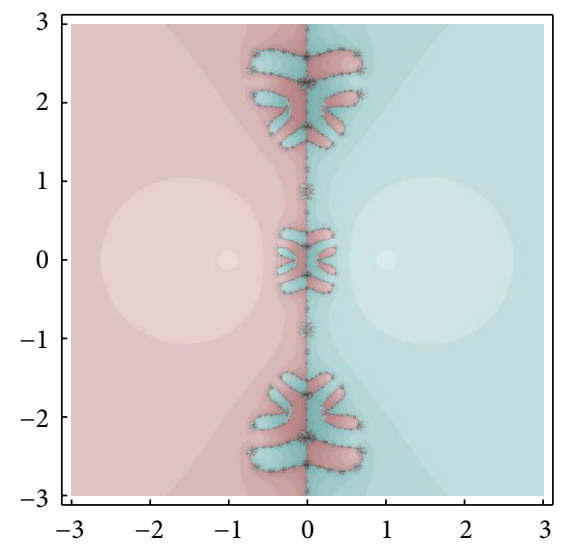

(f)

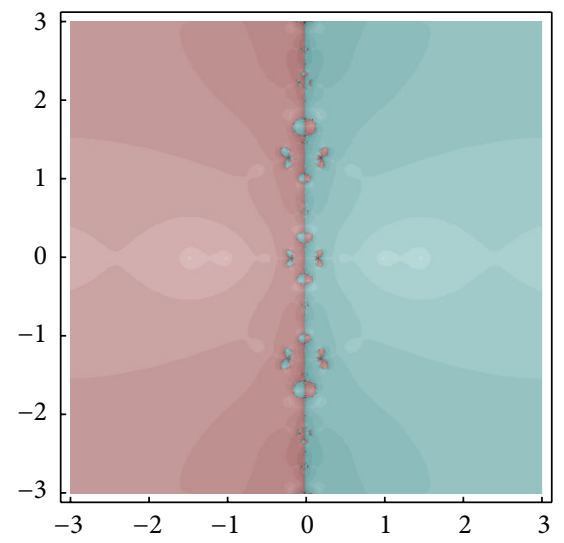

(i)

Figure 8: (a) Kan, (b) Sol, (c) Zhou, (d) Y1, (e) Y2, (f) Y3, (g) Y4, (h) Y5, and (i) Y6, for the roots of the polynomial $P_{4}(z)=\left(z^{2}-1\right)^{5}$.

method Sol has shown best AVG and TDIV. We find that Zhou shows the best result for CPU, while Y 6 shows the worst one.

\section{Conclusion}

We have developed a triparametric family of optimal fourthorder methods and investigated their complex dynamics via Möbius conjugacy map applied to a polynomial of the form
$f(z)=[(z-A)(z-B)]^{m}$ along with the stability analysis of strange fixed points. Besides, different complex dynamics have been also investigated from a viewpoint of extraneous fixed points of the proposed methods (5) when applied to a prototype quadratic polynomial $f(z)=\left(z^{2}-1\right)^{m}$ raised to the power of known multiplicity $m$. To extract information on better initial guesses of an iterative method, we need to carefully examine the basins of attraction. In this paper, we have illustrated a number of basins of attraction for the listed 
TABle 7: Typical Example 1 with $P_{1}(z)=\left(z^{3}-z\right)^{2}, m=2$.

\begin{tabular}{lcccc}
\hline Method & CPU & TCON & AVG & TDIV \\
\hline Kan & 205.469 & 360,000 & 6.20867 & 0 \\
Sol & 111.884 & 351,220 & 5.88726 & 8780 \\
Zhou & 76.331 & 360,000 & 7.34681 & 0 \\
Y1 & 184.503 & 360,000 & 6.03626 & 0 \\
Y2 & 195.422 & 360,000 & 6.22663 & 0 \\
Y3 & 200.024 & 360,000 & 6.34617 & 0 \\
Y4 & 118.436 & 360,000 & 6.35492 & 0 \\
Y5 & 308.039 & 360,000 & 9.56036 & 0 \\
Y6 & 488.376 & 360,000 & 13.786 & 0 \\
\hline
\end{tabular}

TABLE 8: Typical Example 1 with $P_{2}(z)=\left(z^{3}+5 z-7\right)^{3}, m=3$.

\begin{tabular}{lcccc}
\hline Method & CPU & TCON & AVG & TDIV \\
\hline Kan & 442.013 & 360,000 & 5.73999 & 0 \\
Sol & 296.418 & 359,898 & 5.57455 & 102 \\
Zhou & 84.599 & 359,996 & 6.72502 & 4 \\
Y1 & 357.679 & 360,000 & 5.44998 & 0 \\
Y2 & 397.303 & 360,000 & 5.84558 & 0 \\
Y3 & 411.795 & 360,000 & 6.03896 & 0 \\
Y4 & 423.153 & 360,000 & 6.01186 & 0 \\
Y5 & 206.343 & 360,000 & 5.35253 & 0 \\
Y6 & 973.993 & 360,000 & 13.2845 & 0 \\
\hline
\end{tabular}

TABLE 9: Typical Example 1 with $P_{3}(z)=\left(z^{2}+3 z+5\right)^{4}, m=4$.

\begin{tabular}{lcccc}
\hline Method & CPU & TCON & AVG & TDIV \\
\hline Kan & 201.023 & 359,594 & 5.54699 & 406 \\
Sol & 145.612 & 360,000 & 5.36095 & 0 \\
Zhou & 54.226 & 360,000 & 6.00988 & 0 \\
Y1 & 176.749 & 360,000 & 5.32411 & 0 \\
Y2 & 187.17 & 360,000 & 5.58189 & 0 \\
Y3 & 189.401 & 359,998 & 5.66574 & 2 \\
Y4 & 198.277 & 359,725 & 5.73911 & 275 \\
Y5 & 189.385 & 359,996 & 7.25579 & 4 \\
Y6 & 384.09 & 359,994 & 10.9976 & 6 \\
\hline
\end{tabular}

optimal fourth-order iterative methods when applied to various complex polynomials as well as statistical analysis featuring data for CPU time and other tabulated numbers for convergence behavior. From the area of convergence on the basins of attraction, more effective information can be obtained for selecting stable initial values of the iterative methods under consideration. As a future research work, we will devote ourselves to developing a higher-order family of iterative methods along with statistical data analysis as well as an illustrative investigation of the desired basins of attraction.

\section{Conflict of Interests}

The authors declare that there is no conflict of interests regarding the publication of this paper.
TABle 10: Typical Example 1 with $P_{4}(z)=\left(z^{2}-z\right)^{6}, m=6$.

\begin{tabular}{lcccc}
\hline Method & CPU & TCON & AVG & TDIV \\
\hline Kan & 331.018 & 359,994 & 5.76332 & 6 \\
Sol & 235.64 & 359,998 & 5.56602 & 2 \\
Zhou & 55.615 & 360,000 & 6.45457 & 0 \\
Y1 & 251.661 & 360,000 & 5.70224 & 0 \\
Y2 & 252.815 & 360,000 & 5.78377 & 0 \\
Y3 & 253.236 & 360,000 & 5.79558 & 0 \\
Y4 & 252.316 & 360,000 & 5.78629 & 0 \\
$Y 5$ & 267.401 & 360,000 & 8.74116 & 0 \\
$Y 6$ & 416.866 & 359,891 & 8.59493 & 109 \\
\hline
\end{tabular}

TABLE 11: Typical Example 1 with $P_{5}(z)=\left(z^{2}-1\right)^{2}, m=2$.

\begin{tabular}{lcccc}
\hline Method & CPU & TCON & AVG & TDIV \\
\hline Kan & 60.231 & 360,000 & 5.28004 & 0 \\
Sol & 48.375 & 350,408 & 5.00397 & 9592 \\
Zhou & 39.952 & 360,000 & 5.92232 & 0 \\
Y1 & 61.948 & 360,000 & 5.31739 & 0 \\
Y2 & 46.723 & 360,000 & 5.38721 & 0 \\
Y3 & 60.481 & 360,000 & 5.07764 & 0 \\
Y4 & 62.557 & 360,000 & 5.31739 & 0 \\
Y5 & 464.556 & 360,000 & 13.8001 & 0 \\
Y6 & 158.309 & 360,000 & 13.8001 & 0 \\
\hline
\end{tabular}

TABLE 12: Typical Example 1 with $P_{6}(z)=\left(z^{2}-1\right)^{5}, m=5$.

\begin{tabular}{lcccc}
\hline Method & CPU & TCON & AVG & TDIV \\
\hline Kan & 101.743 & 360,000 & 5.45698 & 0 \\
Sol & 75.38 & 360,000 & 5.26937 & 0 \\
Zhou & 37.472 & 360,000 & 5.92232 & 0 \\
Y1 & 119.169 & 360,000 & 5.59266 & 0 \\
Y2 & 119.84 & 360,000 & 5.58321 & 0 \\
Y3 & 96.877 & 360,000 & 5.33871 & 0 \\
Y4 & 100.932 & 360,000 & 5.59266 & 0 \\
Y5 & 150.634 & 360,000 & 8.93996 & 0 \\
Y6 & 164.113 & 359,891 & 8.93996 & 0 \\
\hline
\end{tabular}

\section{Acknowledgment}

The corresponding author (Y. H. Geum) was supported by Basic Science Research Program through the National Research Foundation of Korea funded by the Ministry of Education under the research grant (Project no. 2015R1D1A3A-01020808).

\section{References}

[1] J. A. Wright, J. H. Deane, M. Bartuccelli, and G. Gentile, "Basins of attraction in forced systems with time-varying dissipation," Communications in Nonlinear Science and Numerical Simulation, vol. 29, no. 1-3, pp. 72-87, 2015.

[2] I. K. Argyros and Á. Magreñán, "On the convergence of an optimal fourth-order family of methods and its dynamics," 
Applied Mathematics and Computation, vol. 252, pp. 336-346, 2015.

[3] F. Chicharro, A. Cordero, J. M. Gutiérrez, and J. R. Torregrosa, "Complex dynamics of derivative-free methods for nonlinear equations," Applied Mathematics and Computation, vol. 219, no. 12, pp. 7023-7035, 2013.

[4] B. Neta and C. Chun, "Basins of attraction for several optimal fourth order methods for multiple roots," Mathematics and Computers in Simulation, vol. 103, pp. 39-59, 2014.

[5] S. Kumar, V. Kanwar, and S. Singh, "On some modified families of multipoint iterative methods for multiple roots of nonlinear equations," Applied Mathematics and Computation, vol. 218, no. 14, pp. 7382-7394, 2012.

[6] L. Shengguo, L. Xiangke, and C. Lizhi, "A new fourth-order iterative method for finding multiple roots of nonlinear equations," Applied Mathematics and Computation, vol. 215, no. 3, pp. 12881292, 2009.

[7] S. G. Li, L. Z. Cheng, and B. Neta, "Some fourth-order nonlinear solvers with closed formulae for multiple roots," Computers \& Mathematics with Applications, vol. 59, no. 1, pp. 126-135, 2010.

[8] V. Kanwar, S. Bhatia, and M. Kansal, "New optimal class of higher-order methods for multiple roots, permitting $f^{\prime}\left(x_{n}\right)=$ 0," Applied Mathematics and Computation, vol. 222, no. 1, pp. 564-574, 2013.

[9] L. B. Rall, "Convergence of the Newton process to multiple solutions," Numerische Mathematik, vol. 9, no. 1, pp. 23-37, 1966.

[10] A. M. Ostrowski, Solutions of Equations and System of Equations, Academic Press, New York, NY, USA, 1960.

[11] M. S. Petković, B. Neta, L. D. Petković, and J. Džunić, Multipoint Methods for Solving Non-Linear Equations, Academic Press, 2012.

[12] J. R. Sharma and R. Sharma, "Modified Jarratt method for computing multiple roots," Applied Mathematics and Computation, vol. 217, no. 2, pp. 878-881, 2010.

[13] F. Soleymani, D. K. Babajee, and T. Lotfi, "On a numerical technique for finding multiple zeros and its dynamic," Journal of the Egyptian Mathematical Society, vol. 21, no. 3, pp. 346-353, 2013.

[14] X. Zhou, X. Chen, and Y. Song, "Constructing higher-order methods for obtaining the multiple roots of nonlinear equations," Journal of Computational and Applied Mathematics, vol. 235, no. 14, pp. 4199-4206, 2011.

[15] A. Cordero, J. García-Maimó, J. R. Torregrosa, M. P. Vassileva, and P. Vindel, "Chaos in King's iterative family," Applied Mathematics Letters, vol. 26, no. 8, pp. 842-848, 2013.

[16] T. de Carvalho and M. A. Teixeira, "Basin of attraction of a cuspfold singularity in 3D piecewise smooth vector fields," Journal of Mathematical Analysis and Applications, vol. 418, no. 1, pp.11-30, 2014.

[17] S. Amat, S. Busquier, and S. Plaza, "Dynamics of the King and Jarratt iterations," Aequationes Mathematicae, vol. 69, no. 3, pp. 212-223, 2005.

[18] L. V. Ahlfors, Complex Analysis, McGraw-Hill Book, 1979.

[19] H. T. Kung and J. F. Traub, "Optimal order of one-point and multipoint iteration," Journal of the ACM, vol. 21, no. 4, pp. 643651, 1974.

[20] V. Kanwar, S. Bhatia, and M. Kansal, "New optimal class of higher-order methods for multiple roots, permitting $f^{\prime}\left(x_{n}\right)=$ 0," Applied Mathematics and Computation, vol. 222, pp. 564574, 2013.
[21] F. Soleymani and D. K. R. Babajee, "Computing multiple zeros using a class of quartically convergent methods," Alexandria Engineering Journal, vol. 52, no. 3, pp. 531-541, 2013.

[22] Y. H. Geum and Y. I. Kim, "A two-parameter family of fourthorder iterative methods with optimal convergence for multiple zeros," Journal of Applied Mathematics, vol. 2013, Article ID 369067, 7 pages, 2013.

[23] Y. I. Kim and Y. H. Geum, "A new biparametric family of twopoint optimal fourth-order multiple-root finders," Journal of Applied Mathematics, vol. 2014, Article ID 737305, 7 pages, 2014.

[24] J. Traub, Iterative Methods for the Solution of Equations, Chelsea, 1997.

[25] S. Wolfram, The Mathematica Book, Wolfram Media, 5th edition, 2003.

[26] A. F. Beardon, Iteration of Rational Functions, Springer, New York, NY, USA, 1991.

[27] P. Blanchard, "The dynamics of Newton's method," Proceedings of Symposia in Applied Mathematics, vol. 49, pp. 139-154, 1994.

[28] R. Behl, A. Cordero, S. S. Motsa, and J. R. Torregrosa, "On developing fourth-order optimal families of methods for multiple roots and their dynamics," Applied Mathematics and Computation, vol. 265, pp. 520-532, 2015.

[29] A. A. Magrenan, A. Cordero, J. M. Gutierrez, and J. R. Torregrosa, "Real qualitative behavior of a fourth-order family of iterative methods by using the convergence plane," Mathematics and Computers in Simulation, vol. 105, pp. 49-61, 2014.

[30] A. Cordero, C. Jordán, and J. Torregrosa, "One-point Newtontype iterative methods: a unified point of view," Journal of Computational and Applied Mathematics, vol. 275, pp. 366-374, 2015.

[31] B. Kalantari and Y. Jin, "On extraneous fixed-points of the basic family of iteration functions," BIT Numerical Mathematics, vol. 43, no. 2, pp. 453-458, 2003.

[32] E. R. Vrscay and W. J. Gilbert, "Extraneous fixed points, basin boundaries and chaotic dynamics for Schröder and König rational iteration functions," Numerische Mathematik, vol. 52, no. 1, pp. 1-16, 1988.

[33] E. Schröder, "Über unendlich viele algorithmen zur auflösung der gleichungen," Mathematische Annalen, vol. 2, pp. 317-365, 1870.

[34] A. Cayley, "Application of the Newton-Fourier method to an imaginary root of an equation," The Quarterly Journal of Pure and Applied Mathematics, vol. 16, pp. 179-185, 1879.

[35] M. Scott, B. Neta, and C. Chun, "Basin attractors for various methods," Applied Mathematics and Computation, vol. 218, no. 6, pp. 2584-2599, 2011.

[36] B. Neta, M. Scott, and C. Chun, "Basins of attraction for several methods to find simple roots of nonlinear equations," Applied Mathematics and Computation, vol.218, no. 21, pp. 10548-10556, 2012. 


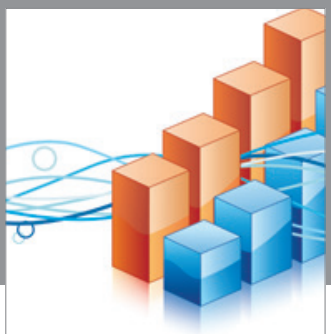

Advances in

Operations Research

vatem alat4

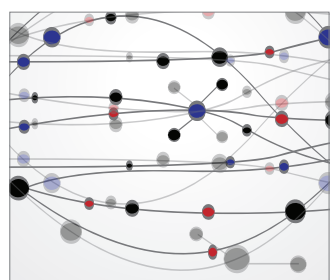

\section{The Scientific} World Journal
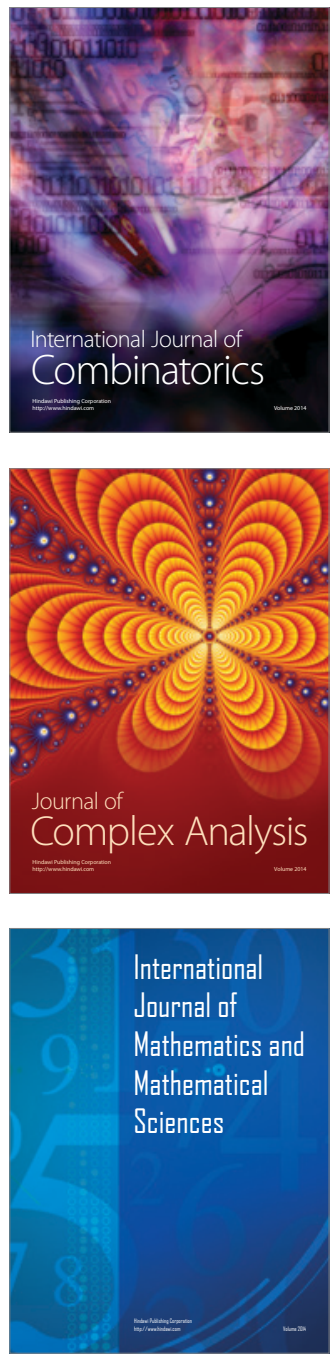
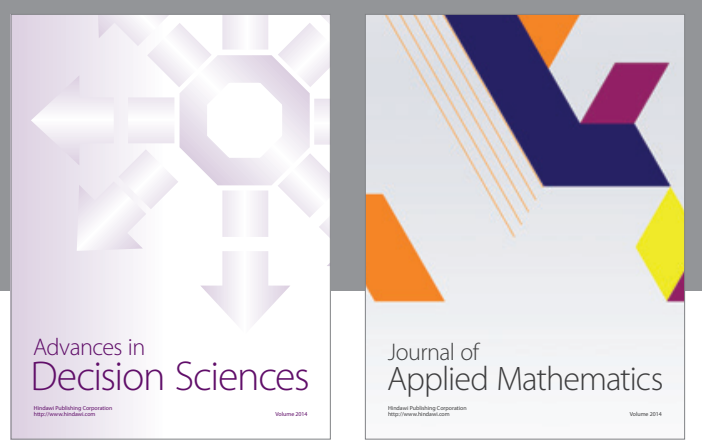

Algebra

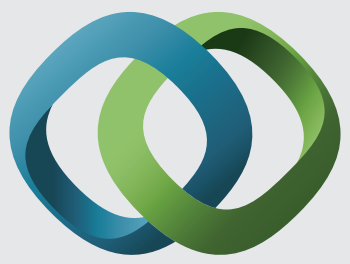

\section{Hindawi}

Submit your manuscripts at

http://www.hindawi.com
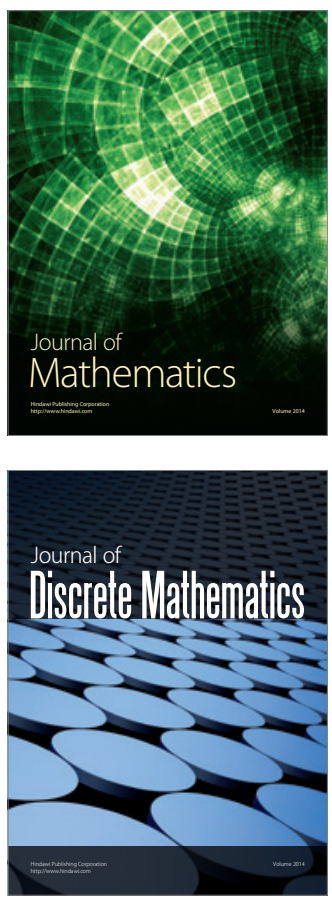

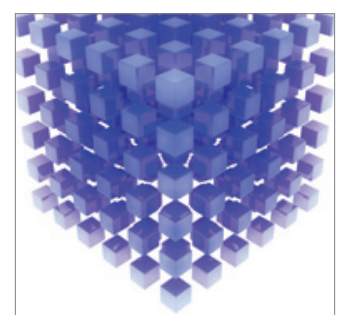

Mathematical Problems in Engineering
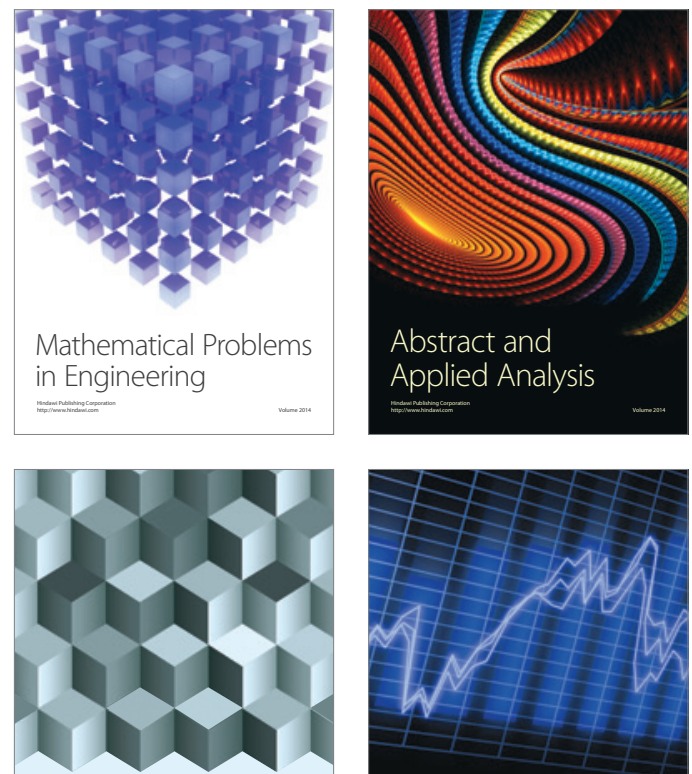

Journal of

Function Spaces

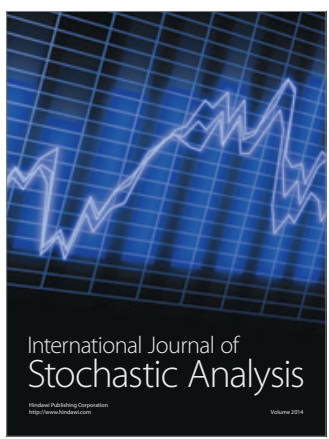

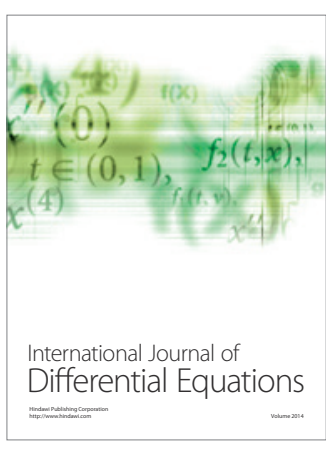
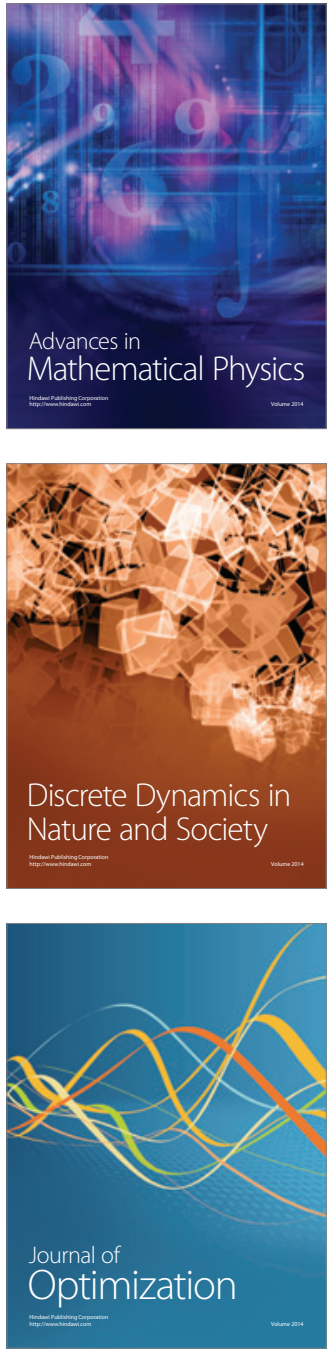